\title{
Investigating One-, Two-, and Triple-Wave Solutions via Multiple Exp-Function Method Arising in Engineering Sciences
}

\author{
Pengbo Wan $\left(\mathbb{D},{ }^{1}\right.$ Jalil Manafian $\mathbb{D}^{2},{ }^{2}$ Hajar Farhan Ismael $\mathbb{D}^{3},{ }^{3}$ and Sizar Abid Mohammed $\mathbb{D}^{4}$ \\ ${ }^{1}$ Department of Finance, Economics and Management School of Wuhan University, Wuhan City, Hubei Province, China \\ ${ }^{2}$ Department of Applied Mathematics, Faculty of Mathematical Sciences, University of Tabriz, Tabriz, Iran \\ ${ }^{3}$ Department of Mathematics, Faculty of Science, University of Zakho, Zakho, Iraq \\ ${ }^{4}$ Department of Mathematics, College of Basic Education, University of Duhok, Zakho Street 38, 1006 AJ Duhok, Iraq
}

Correspondence should be addressed to Jalil Manafian; j_manafianheris@tabrizu.ac.ir

Received 28 January 2020; Accepted 22 April 2020; Published 8 June 2020

Academic Editor: Ziemowit Popowicz

Copyright (c) 2020 Pengbo Wan et al. This is an open access article distributed under the Creative Commons Attribution License, which permits unrestricted use, distribution, and reproduction in any medium, provided the original work is properly cited.

\begin{abstract}
The multiple Exp-function method is employed for searching the multiple soliton solutions for the new extended $(3+1)$ dimensional Jimbo-Miwa-like (JM) equation, the extended $(2+1)$-dimensional Calogero-Bogoyavlenskii-Schiff (eCBS) equation, the generalization of the $(2+1)$-dimensional Bogoyavlensky-Konopelchenko (BK) equation, and a variablecoefficient extension of the DJKM (vDJKM) equation, which contain one-soliton-, two-soliton-, and triple-soliton-kind solutions. The physical phenomena of these gained multiple soliton solutions are analyzed and indicated in figures by selecting suitable values.
\end{abstract}

\section{Introduction}

Many nonlinear physical phenomena arise in various fields of engineering and science such as fluid dynamics, nuclear reactor dynamics, hydrodynamics, molecular biology, quantum mechanics, plasma physics, optical fibres, and solid state physics. To describe these complex physical phenomena, nonlinear differential equations play a significant role. Therefore, obtaining the solutions of these nonlinear equations is a topic of great interest in the study of many fields of science. To better understand the working of a physical problem, a mathematical model was brought into the picture in the form of nonlinear PDEs. The solutions of partial differential equations give the detailed summary about the nature of phenomena involved. Many numerical and analytical methods have been derived to deal with these kinds of scientific problems. We need to adopt an effective and powerful method to investigate such type of mathematical model which gives the solutions upholding physical reality. In most of the analytic techniques, linearization of the system is the main topic to focus on, and also, it is assumed that the nonlinearities are relatively insignificant. Sometimes, these assumptions make a strong effect on the solutions in respect to the real physics of the phenomena involved. Thus, finding the solutions of nonlinear ODEs and PDEs is still a significant problem. For this, we need new techniques to develop approximate and exact solutions. Several analytical and numerical techniques have been formulated for tackling these types of nonlinear models, including the Exp-function method $[1,2]$, the homotopy analysis method [3], the homotopy perturbation method [4], the $\left(G^{\prime} / G\right)$-expansion method [5], the improved $\tan (\phi / 2)$-expansion method ([6-8]), the Hirota bilinear method ([9-13]), the He variational principle [14, 15], the binary Darboux transformation [16], the Lie group analysis $[17,18]$, and the Bäcklund transformation method [19]. Moreover, many powerful methods have been used to investigate the new properties of mathematical models which are symbolizing serious real world problems $([20-24])$. This 
study is aimed at investigating the following four forms of nonlinear PDEs:

(i) Extended $(3+1)$-dimensional Jimbo-Miwa-like (JM) equations $([25-34])$ is given as

$$
\begin{aligned}
\Psi_{x x x y} & +3 \Psi_{x x} \Psi_{y}+3 \Psi_{x} \Psi_{x y}+2 \Psi_{y t} \\
& -3\left(\Psi_{x z}+\Psi_{y z}+\Psi_{z z}\right)=0
\end{aligned}
$$

and

$$
\begin{aligned}
\Psi_{x x x y} & +3 \Psi_{x x} \Psi_{y}+3 \Psi_{x} \Psi_{x y}-3 \Psi_{x z} \\
& +2\left(\Psi_{x t}+\Psi_{y t}+\Psi_{z t}\right)=0
\end{aligned}
$$

explains some $(3+1)$-dimensional waves in physics $[25,26]$, in which $\Psi(x, y, z, t)$ is a function including spaces $x, y, z$ and time $t$. In addition, this equation cannot pass certain conventional integrability tests [27]. Qi et al. [32] studied the extended $(3+1)$ dimensional Jimbo-Miwa-like equation via the Hirota method and obtained the solitary-wave solutions and novel exact soliton solutions.

(ii) The extended (2+1)-dimensional CalogeroBogoyavlenskii-Schiff (eCBS) equation [35] is given as

$$
\begin{aligned}
\Psi_{x t} & +\Psi_{x x x y}+6 \Psi_{x x} \Psi_{y}+6 \Psi_{x} \Psi_{x y}+\delta_{1} \Psi_{x y} \\
& +\delta_{2} \Psi_{x x}+\delta_{3}\left(\Psi_{x x x x}+12 \Psi_{x} \Psi_{x x}\right)=0
\end{aligned}
$$

where $\delta_{1}, \delta_{2}$, and $\delta_{3}$ are arbitrary values. The eCBS equation will be changed to a $(2+1)$-dimensional CBS equation when $\delta_{1}=\delta_{2}=\delta_{3}=0$ [36]. Ren et al. [35] investigated the extended $(2+1)$-dimensional Calogero-Bogoyavlenskii-Schiff-like equation by using the generalized bilinear operators based on a prime number $p=3$.

(iii) The generalization of the $(2+1)$-dimensional Bogoyavlensky-Konopelchenko (BK) equation [37] is given as

$$
\begin{aligned}
\Psi_{x t} & +\alpha\left(\Psi_{x x x x}+6 \Psi_{x} \Psi_{x x}\right)+\beta\left(\Psi_{x x x y}+3 \Psi_{x x} \Psi_{y}\right. \\
& \left.+3 \Psi_{x} \Psi_{x y}\right)+\delta_{1} \Psi_{x x}+\delta_{2} \Psi_{x y}+\delta_{3} \Psi_{y y}=0
\end{aligned}
$$

The $(3+1)$-dimensional VC B-type $\mathrm{KP}$ equation is extended from the KP equation and can explain some notable $(3+1)$-dimensional waves in fluid dynamics [37]. Also, Zhang and Pang [38] found the lump and lump-type solutions through the Hirota bilinear form for the variable-coefficient B-type Kadomtsev-Petviashvili equation. (iv) The variable-coefficient extension of the DJKM (vDJKM) equation ([39-48]) is given as

$$
\begin{aligned}
\Psi_{x x x x y} & +4 \Psi_{x} \Psi_{x x y}+2 \Psi_{y} \Psi_{x x x}+6 \Psi_{x x} \Psi_{x y} \\
& -\alpha \Psi_{y y y}-2 \beta g(t) \Psi_{x x t}+h(t) \Psi_{x x y}=0,
\end{aligned}
$$

where the wave amplitude $u(x, y, t)$ is a function of variables and $x, y, t, g(t)$, and $h(t)$ are functions of $t$. The $\mathrm{vD}$ JKM equation (6) reduces to the DJKM equation when $g(t)=1$ and $h(t)=0$ as follows:

$$
\begin{aligned}
\Psi_{x x x x y} & +4 \Psi_{x} \Psi_{x x y}+2 \Psi_{y} \Psi_{x x x} \\
& +6 \Psi_{x x} \Psi_{x y}-\alpha \Psi_{y y y}-2 \beta \Psi_{x x t}=0 .
\end{aligned}
$$

The soliton solutions to a few $(3+1)$-dimensional generalized nonlinear integrable equations have been constructed. The adopted approach is the multiple Exp-function method, which was presented earlier in the valuable work by Ma et al. [49]. Recently, special kinds of reductions of soliton solutions to rational functions that are being actively studied are lump solutions to nonlinear partial differential equations by $\mathrm{Ma}$ and Zhou [50] and their interactions with solitons to the Hirota-Satsuma-Ito equation in $(2+1)$-dimensions by $\mathrm{Ma}$ [51], even for linear PDEs by the same author [52]. Cordero et al. constructed the stability analysis of the fourth-order iterative method for finding multiple roots of nonlinear equations [53]. Gao et al. obtained the optical soliton solutions of the cubic-quartic nonlinear Schrödinger and resonant nonlinear Schrödinger equations with the parabolic law [54]. The truncated Painlevé expansion was employed to derive a Bäcklund transformation of a $(2+1)$-dimensional nonlinear system by Zhao and Han [55]. Also, Gao et al. studied on the conformable $(2+1)$-dimensional Ablowitz-KaupNewell-Segur equation in order to show the existence of complex combined dark-bright soliton solutions [56]. The same authors presented the nonlinear Zoomeron equation by using the newly extended direct algebraic technique [57]. The instability modulation for the $(2+1)$ dimension paraxial wave equation and its new optical soliton solutions in Kerr's media by utilizing the modified auxiliary expansion method have been studied in [58]. In [59], the authors found new complex solitons to the perturbed nonlinear Schrödinger model with the help of an analytical method. In this paper, we will study the multiple Exp-function method for determining the multiple soliton solutions. The multiple Exp-function method used by some of the powerful authors for various nonlinear equations include the nonlinear evolution equations [60], the $(2+1)$-dimensional Calogero-Bogoyavlenskii-Schiff equation [61], the generalized $(1+1)$-dimensional and $(2+1)$-dimensional Ito equations [62], a new generalization of the associated Camassa-Holm equation [63], the $(3+1)$-dimensional generalized KP and BKP equations [64], and the new $(2+1)$-dimensional Korteweg-de Vries equation [65]. In [65], Liu et al. utilized the multiple Exp-function method for the most well-known equation, namely, the Korteweg-de Vries (KdV) equation, and gained one-soliton-, two-soliton-, and three-soliton-type 
solutions with interpretations for the obtained soliton solutions. Moreover, they analyzed and illustrated the propagation and interaction of some soliton solutions by selecting appropriate values. Also, the elasticity and being unchanged for some solutions when interacting among three solitons and after the collision were investigated.

The rest of this paper is structured as follows: the multiple Exp-function scheme is summarized in Section 2. In Sections 3-7, the extended JM equations, the extended CBS equation, the generalized $\mathrm{BK}$ equation, and the vDJKM equation, respectively, will be investigated to find one-soliton, two-soliton, and triple-soliton solutions. In the last section, the conclusions are given.

\section{Multiple Exp-Function Method}

This section elucidates a systematic explanation of the multiple Exp-function method [60-64] so that it can be further applied to the nonlinear PDEs in order to furnish its exact solutions:

Step 1. The following NLPDE are as follows:

$$
\mathcal{N}\left(x, y, t, \Psi, \Psi_{x}, \Psi_{y}, \Psi_{z}, \Psi_{t}, \Psi_{x x}, \Psi_{t t}, \cdots\right)=0
$$

We commence a sequence of novel variables $\xi_{i}=$ $\xi_{i}(x, y, z, t), 1 \leq i \leq n$, by solvable PDEs, for example, the linear ones:

$$
\begin{gathered}
\xi_{i, x}=\alpha_{i} \xi_{i}, \\
\xi_{i, y}=\beta_{i} \xi_{i}, \\
\xi_{i, z}=\gamma_{i} \xi_{i}, \\
\xi_{i, t}=\delta_{i} \xi_{i}, \\
1 \leq i \leq n,
\end{gathered}
$$

where $\alpha_{i}, \beta_{i}, \gamma_{i}, 1 \leq i \leq n$ are the angular wave numbers and $\delta_{i}, 1 \leq i \leq n$ are the wave frequencies. It must be pointed out that this is frequently the initiating step for constructing the exact solutions to nonlinear partial differential equations; moreover, solving such linear equations redounds to the exponential function solutions,

$$
\xi_{i}=\omega_{i} e^{\theta_{i}}, \quad \theta_{i}=\alpha_{i} x+\beta_{i} y+\gamma_{i} z-\delta_{i} t, 1 \leq i \leq n,
$$

in which $\omega_{i}, 1 \leq i \leq n$, are undetermined values.

Step 2. Supposing the solution of equation (7) happens to be of the following form in terms of new variables $\xi_{i}, 1 \leq i \leq n$ :

$$
\begin{aligned}
\Psi(x, y, z, t)=\frac{\Delta\left(\xi_{1}, \xi_{2}, \cdots, \xi_{n}\right)}{\Omega\left(\xi_{1}, \xi_{2}, \cdots, \xi_{n}\right)}, \\
\Delta=\sum_{r, s=1}^{n} \sum_{i, j=0}^{M} \Delta_{r s, i j} \xi_{r}^{i} \xi_{s}^{j}, \Omega=\sum_{r, s=1}^{n} \sum_{i, j=0}^{N} \Omega_{r s, i j} \xi_{r}^{i} \xi_{s}^{j},
\end{aligned}
$$

in which $\Delta_{r s, i j}$ and $\Omega_{r s, i j}$ are values to be settled. Plugging equation (10) into equation (7) and ordering the numerator of the rational function to zero, we can gain a series of the nonlinear algebraic equations about the variables $\alpha_{i}, \beta_{i}, \gamma_{i}$, $\delta_{i}, \Delta_{r s, i j}$ and $\Omega_{r s, i j}$. Solving the solutions for these nonlinear algebraic equations and inserting these solutions into equation (10), the multiple soliton solutions for equation (7) can be achieved as follows:

$$
\Psi(x, y, z, t)=\frac{\Delta\left(\omega_{1} e^{\alpha_{1} x+\beta_{1} y+\gamma_{1} x-\delta_{1} t}, \cdots, \omega_{n} e^{\alpha_{n} x+\beta_{n} y+\gamma_{n} x-\delta_{n} t}\right)}{\Omega\left(\omega_{1} e^{\alpha_{1} x+\beta_{1} y+\gamma_{1} x-\delta_{1} t}, \cdots, \omega_{n} e^{\alpha_{n} x+\beta_{n} y+\gamma_{n} x-\delta_{n} t}\right)},
$$

and also we have

$$
\begin{aligned}
& \Delta_{t}=\sum_{i=1}^{n} \Delta_{\xi_{i}} \xi_{i, t}, \\
& \Omega_{t}=\sum_{i=1}^{n} \Omega_{\xi_{i}} \xi_{i, t}, \\
& \Delta_{x}=\sum_{i=1}^{n} \Delta_{\xi_{i}} \xi_{i, x} \\
& \Omega_{x}=\sum_{i=1}^{n} \Omega_{\xi_{i}} \xi_{i, x} \\
& \Delta_{y}=\sum_{i=1}^{n} \Delta_{\xi_{i}} \xi_{i, y}, \\
& \Omega_{y}=\sum_{i=1}^{n} \Omega_{\xi_{i}} \xi_{i, y}, \\
& \Delta=\Delta\left(\xi_{1}, \xi_{2}, \cdots, \xi_{n}\right), \\
& \Omega=\Omega\left(\xi_{1}, \xi_{2}, \cdots, \xi_{n}\right), \\
& \Delta_{\xi_{i}}=\frac{d \Delta}{d_{\xi_{i}}}, \\
& \Omega_{\xi_{i}}=\frac{d \Omega}{d_{\xi_{i}}}, \\
& \xi_{i, t}=\frac{d \xi_{i}}{d t}, \\
& \xi_{i, x}=\frac{d \xi_{i}}{d x}, \\
& \xi_{i, y}=\frac{d \xi_{i}}{d y} \\
& \Psi_{t}=\frac{\Omega \sum_{i=1}^{n} \Delta_{\xi_{i}} \xi_{i, t}-\Delta \sum_{i=1}^{n} \Omega_{\xi_{i}} \xi_{i, t}}{\Omega^{2}}, \\
& \Psi_{x}=\frac{\Omega \sum_{i=1}^{n} \Delta_{\xi_{i}} \xi_{i, x}-\Delta \sum_{i=1}^{n} \Omega_{\xi_{i}} \xi_{i, x}}{\Omega^{2}}, \\
& \Psi_{y}=\frac{\Omega \sum_{i=1}^{n} \Delta_{\xi_{i}} \xi_{i, y}-\Delta \sum_{i=1}^{n} \Omega_{\xi_{i}} \xi_{i, y}}{\Omega^{2}}, \\
& \Psi_{z}=\frac{\Omega \sum_{i=1}^{n} \Delta_{\xi_{i}} \xi_{i, z}-\Delta \sum_{i=1}^{n} \Omega_{\xi_{i}} \xi_{i, z}}{\Omega^{2}} .
\end{aligned}
$$




\section{Multiple Soliton Solutions for the Extended $(3+1)$ JM Equation (1)}

3.1. Set I: One-Wave Solution. We commence with a onewave function based on the statement in Step 2 in the previous section; we suppose that equation (1) has the rational function of the one-wave solution as shown in the following form:

$$
\begin{aligned}
\Psi(x, y, z, t)=\frac{\Delta_{1}}{\Omega_{1}}, \quad \Omega_{1} & =1+\rho_{1} e^{\alpha_{1} x+\beta_{1} y+\gamma_{1} z-\delta_{1} t}, \\
\Delta_{1} & =\sigma_{1}+\rho_{1} \sigma_{2} e^{\alpha_{1} x+\beta_{1} y+\gamma_{1} z-\delta_{1} t},
\end{aligned}
$$

in which $\sigma_{1}$ and $\sigma_{2}$ are unfound constants. Plugging (13) into equation (1), we gain to the following case:

$$
\begin{aligned}
& \rho_{1}=\rho_{1}, \\
& \sigma_{1}=\sigma_{1}, \\
& \sigma_{2}=\frac{\rho_{2}\left(2 \alpha_{1} \rho_{1}+\sigma_{1}\right)}{\rho_{1}}, \\
& \delta_{1}=\frac{\alpha_{1}^{3} \beta_{1}-3 \alpha_{1} \gamma_{1}-3 \beta_{1} \gamma_{1}-3 \gamma_{1}^{2}}{2 \beta_{1}} .
\end{aligned}
$$

Therefore, the resulting one-wave solution reads as

$$
\Psi(x, y, z, t)=\frac{\sigma_{1}+\rho_{2}\left(2 \alpha_{1} \rho_{1}+\sigma_{1}\right) e^{\alpha_{1} x+\beta_{1} y+\gamma_{1} z-\left(\left(\alpha_{1}^{3} \beta_{1}-3 \alpha_{1} \gamma_{1}-3 \beta_{1} \gamma_{1}-3 \gamma_{1}^{2}\right) /\left(2 \beta_{1}\right)\right) t}}{1+\rho_{1} e^{\alpha_{1} x+\beta_{1} y+\gamma_{1} z-\left(\left(\alpha_{1}^{3} \beta_{1}-3 \alpha_{1} \gamma_{1}-3 \beta_{1} \gamma_{1}-3 \gamma_{1}^{2}\right) /\left(2 \beta_{1}\right)\right) t}} .
$$

By selecting the suitable values of parameters, the graphic presentation of the periodic wave solution is presented in Figure 1 including the $3 \mathrm{D}$ plot, the contour plot, the density plot, and the $2 \mathrm{D}$ plot when three spaces arise at spaces $x=-1, x=0$, and $x=1$.

3.2. Set II: Two-Wave Solutions. We commence with the twowave functions based on the statement in Step 2 in the previous section; we suppose that equation (1) has the rational function of two-wave solutions as shown in the following form:

$$
\begin{aligned}
\Psi(x, y, z, t)= & \frac{\Delta_{2}}{\Omega_{2}}, \\
\Omega_{2}= & +\sigma_{1} e^{\alpha_{1} x+\beta_{1} y+\gamma_{1} z-\delta_{1} t}+\sigma_{2} e^{\alpha_{2} x+\beta_{2} y+\gamma_{2} z-\delta_{2} t} \\
& +\sigma_{1} \sigma_{2} \sigma_{12} e^{\left(\alpha_{1}+\alpha_{2}\right) x+\left(\beta_{1}+\beta_{2}\right) y+\left(\gamma_{1}+\gamma_{2}\right) z-\left(\delta_{1}+\delta_{2}\right) t}, \\
\Delta_{2}= & \rho_{1} e^{\alpha_{1} x+\beta_{1} y+\gamma_{1} z-\delta_{1} t}+\rho_{2} e^{\alpha_{2} x+\beta_{2} y+\gamma_{2} z-\delta_{2} t} \\
& +\rho_{1} \rho_{2} \rho_{12} e^{\left(\alpha_{1}+\alpha_{2}\right) x+\left(\beta_{1}+\beta_{2}\right) y+\left(\gamma_{1}+\gamma_{2}\right) z-\left(\delta_{1}+\delta_{2}\right) t}
\end{aligned}
$$

Plugging (16) along with (17) into equation (1), we get to the following case:

$$
\begin{aligned}
\sigma_{1} & =\sigma_{1}, \\
\sigma_{2} & =\sigma_{2}, \\
\delta_{1} & =\frac{1}{2} \alpha_{1}^{3}, \\
\delta_{2} & =\frac{1}{2} \alpha_{2}^{3}, \\
\rho_{1} & =2 \alpha_{1} \sigma_{1}, \\
\rho_{2} & =2 \alpha_{2} \sigma_{2}, \\
\rho_{12} & =\frac{\left(\beta_{1}-\beta_{2}\right)\left(\alpha_{1}-\alpha_{2}\right)}{2 \alpha_{1} \alpha_{2}\left(\beta_{1}+\beta_{2}\right)}, \\
\sigma_{12} & =\frac{\left(\beta_{1}-\beta_{2}\right)\left(\alpha_{1}-\alpha_{2}\right)}{\left(\beta_{1}+\beta_{2}\right)\left(\alpha_{1}+\alpha_{2}\right)} .
\end{aligned}
$$

Therefore, the resulting two-wave solution reads as

$$
\Psi_{2}(x, y, z, t)=\frac{2 \alpha_{1} \sigma_{1} e^{\Lambda_{1}}+2 \alpha_{2} \sigma_{2} e^{\Lambda_{2}}+2 \sigma_{1} \sigma_{2}\left(\left(\left(\beta_{1}-\beta_{2}\right)\left(\alpha_{1}-\alpha_{2}\right)\right) /\left(\beta_{1}+\beta_{2}\right)\right) e^{\Lambda_{1}+\Lambda_{2}}}{1+\sigma_{1} e^{\Lambda_{1}}+\sigma_{2} e^{\Lambda_{2}}+\sigma_{1} \sigma_{2}\left(\left(\left(\beta_{1}-\beta_{2}\right)\left(\alpha_{1}-\alpha_{2}\right)\right) /\left(\left(\beta_{1}+\beta_{2}\right)\left(\alpha_{1}+\alpha_{2}\right)\right)\right) e^{\Lambda_{1}+\Lambda_{2}}},
$$

in which $\Lambda_{i}=\alpha_{i} x+\beta_{i} y+\gamma_{i} z-(1 / 2) \alpha_{i}^{3} t, i=1,2$. By selecting the suitable values of parameters, the graphic representation of the periodic wave solution is presented in Figure 2 includ- ing the 3D plot, the contour plot, the density plot, and the $2 \mathrm{D}$ plot when three spaces arise at spaces $x=-10, x=0$, and $x=10$. 

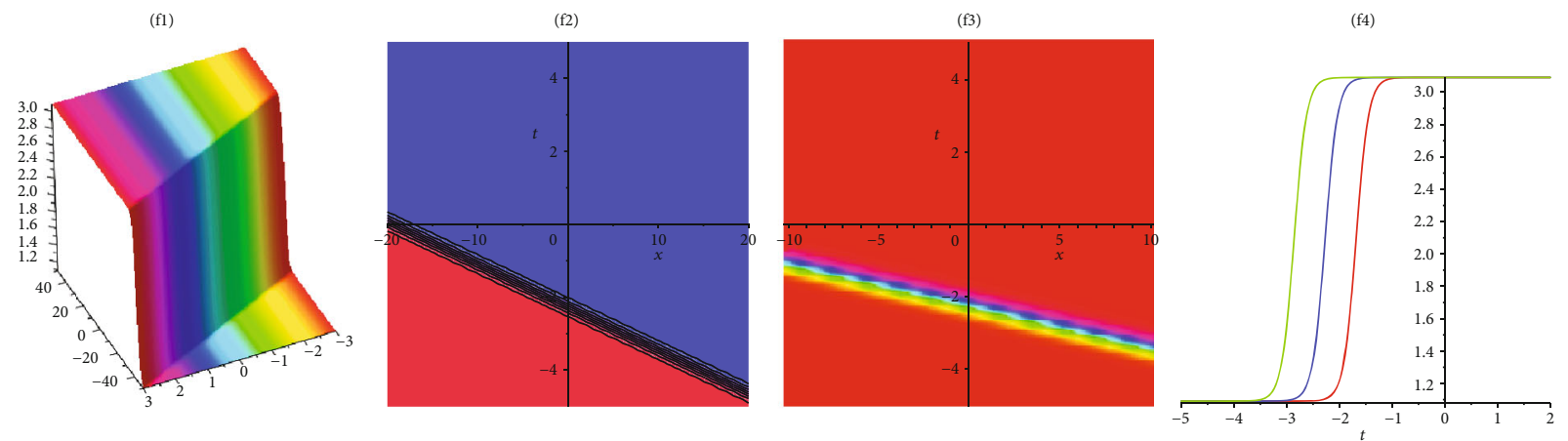

FIgURE 1: The one-wave solution (15) at $\alpha_{1}=1, \beta_{1}=1.5, \sigma_{2}=1.5, \rho_{1}=2.2, \gamma_{1}=2, \rho_{2}=3, y=10$, and $z=2$.
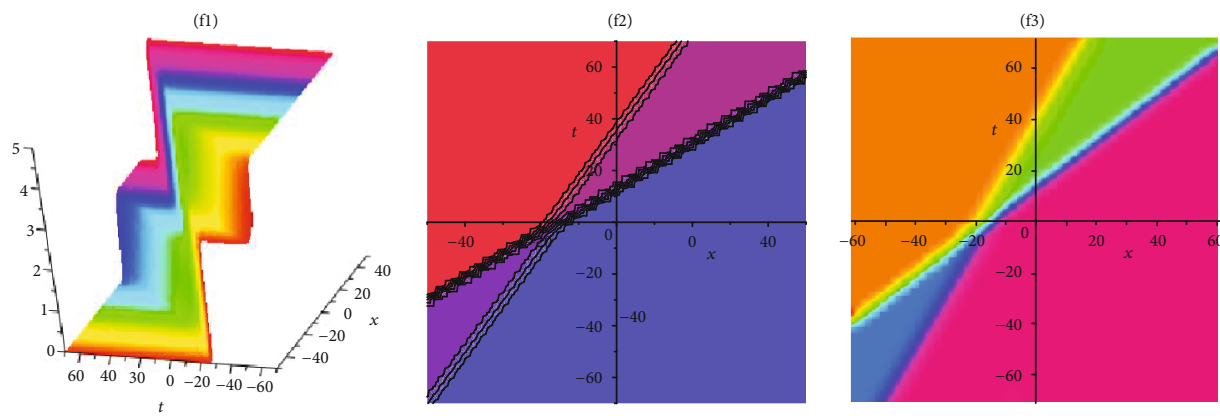

(f4)

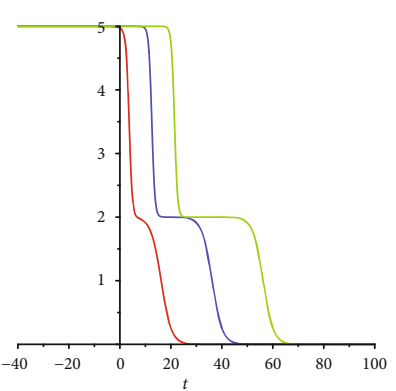

FIGURE 2: The two-wave solution (19) at $\alpha_{1}=1, \alpha_{2}=1.5, \beta_{1}=1.5, \beta_{2}=2, \sigma_{1}=1.1, \sigma_{2}=2, \gamma_{1}=1.5, \gamma_{2}=2, y=10$, and $z=2$.

3.3. Set III: Triple-Wave Solutions. We commence with threewave functions based on the statement in Step 2 in the previous section; we suppose that equation (1) has the rational function of triple-wave solutions as shown in the following form:

$$
\begin{aligned}
\Psi(x, y, z, t)= & \frac{\Delta_{3}}{\Omega_{3}} \\
\Omega_{3}= & +\rho_{1} e^{\Lambda_{1}}+\rho_{2} e^{\Lambda_{2}}+\rho_{3} e^{\Lambda_{3}}+\rho_{1} \rho_{2} \rho_{12} e^{\Lambda_{1}+\Lambda_{2}} \\
& +\rho_{1} \rho_{3} \rho_{13} e^{\Lambda_{1}+\Lambda_{3}}+\rho_{2} \rho_{3} \rho_{23} e^{\Lambda_{2}+\Lambda_{3}} \\
& +\rho_{1} \rho_{2} \rho_{3} \rho_{12} \rho_{13} \rho_{23} e^{\Lambda_{1}+\Lambda_{2}+\Lambda_{3}} \\
\Delta_{3}= & \sigma_{1} e^{\Lambda_{1}}+\sigma_{2} e^{\Lambda_{2}}+\sigma_{3} e^{\Lambda_{3}}+\sigma_{1} \sigma_{2} \sigma_{12} e^{\Lambda_{1}+\Lambda_{2}} \\
& +\sigma_{1} \sigma_{3} \sigma_{13} e^{\Lambda_{1}+\Lambda_{3}}+\sigma_{2} \sigma_{3} \sigma_{23} e^{\Lambda_{2}+\Lambda_{3}} \\
& +\sigma_{1} \sigma_{2} \sigma_{3} \sigma_{12} \sigma_{13} \sigma_{23} e^{\Lambda_{1}+\Lambda_{2}+\Lambda_{3}}
\end{aligned}
$$

in which $\Lambda_{i}=\alpha_{i} x+\beta_{i} y+\gamma_{i} x-\delta_{i} t, i=1,2,3$. Plugging (20) along with (21) into equation (1), we obtain the following case:

$$
\begin{aligned}
\sigma_{i} & =\sigma_{i}, \\
\delta_{i} & =\frac{1}{2} \alpha_{i}^{3}, \\
\rho_{i} & =2 \alpha_{i} \sigma_{i}, \\
i & =1,2,3,
\end{aligned}
$$

$$
\begin{aligned}
& \rho_{12}=\frac{\left(\beta_{1}-\beta_{2}\right)\left(\alpha_{1}-\alpha_{2}\right)}{2 \alpha_{1} \alpha_{2}\left(\beta_{1}+\beta_{2}\right)}, \\
& \sigma_{12}=\frac{\left(\beta_{1}-\beta_{2}\right)\left(\alpha_{1}-\alpha_{2}\right)}{\left(\beta_{1}+\beta_{2}\right)\left(\alpha_{1}+\alpha_{2}\right)}, \\
& \rho_{13}=\frac{\left(\beta_{1}-\beta_{3}\right)\left(\alpha_{1}-\alpha_{3}\right)}{2 \alpha_{1} \alpha_{3}\left(\beta_{1}+\beta_{3}\right)}, \\
& \sigma_{13}=\frac{\left(\beta_{1}-\beta_{3}\right)\left(\alpha_{1}-\alpha_{3}\right)}{\left(\beta_{1}+\beta_{3}\right)\left(\alpha_{1}+\alpha_{3}\right)}, \\
& \rho_{23}=\frac{\left(\beta_{2}-\beta_{3}\right)\left(\alpha_{2}-\alpha_{3}\right)}{2 \alpha_{2} \alpha_{3}\left(\beta_{2}+\beta_{3}\right)}, \\
& \sigma_{23}=\frac{\left(\beta_{2}-\beta_{3}\right)\left(\alpha_{2}-\alpha_{3}\right)}{\left(\beta_{2}+\beta_{3}\right)\left(\alpha_{2}+\alpha_{3}\right)} .
\end{aligned}
$$

Therefore, the resulting three-wave solution reads as

$$
\Psi_{3}(x, y, z, t)=\frac{\Delta_{3}}{\Omega_{3}},
$$

in which $\Delta_{3}$ and $\Omega_{3}$ with their relations are given in (21) and (22). By selecting suitable values of parameters, the graphic representation of the periodic wave solution is presented in Figure 3 including the 3D plot, the contour plot, 

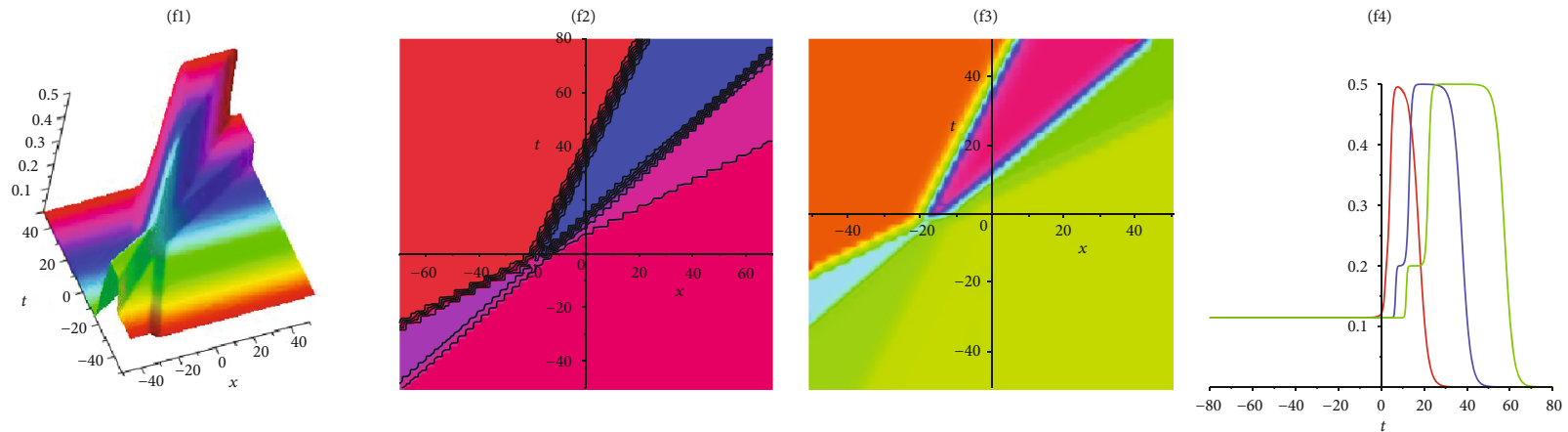

Figure 3: The three-wave solution (23) at $\alpha_{1}=1, \alpha_{2}=1.5, \alpha_{3}=2, \sigma_{1}=1.1, \sigma_{2}=2, \sigma_{3}=2.7, \beta_{1}=2.5, \beta_{2}=2, \beta_{3}=2.6, \gamma_{1}=1.5, \gamma_{2}=2, \gamma_{3}=3$, $y=10$, and $z=2$.

the density plot, and the $2 \mathrm{D}$ plot when three spaces arise at spaces $x=-10, x=0$, and $x=10$.

\section{Multiple Soliton Solutions for the Extended $(3+1)$ JM Equation (2)}

4.1. Set I: One-Wave Solution. We commence with the onewave function based on the statement in Step 2 in the previous section; we suppose that equation (2) has the rational function of the one-wave solution as shown in the following form:

$$
\begin{aligned}
& \Psi(x, y, z, t)=\frac{\Delta_{1}}{\Omega_{1}}, \quad \Omega_{1}=\rho_{1}+\rho_{2} e^{\alpha_{1} x+\beta_{1} y+\gamma_{1} z-\delta_{1} t}, \\
& \Delta_{1}=\sigma_{1}+\sigma_{2} e^{\alpha_{1} x+\beta_{1} y+\gamma_{1} z-\delta_{1} t},
\end{aligned}
$$

in which $\rho_{1}, \rho_{2}, \sigma_{1}$, and $\sigma_{2}$ are unfound constants. Plugging (24) into equation (1), we gain the following case:

$$
\begin{aligned}
& \rho_{1}=\rho_{1}, \\
& \rho_{2}=\rho_{2}, \\
& \sigma_{1}=\sigma_{1}, \\
& \sigma_{2}=\frac{\rho_{2}\left(2 \alpha_{1} \rho_{1}+\sigma_{1}\right)}{\rho_{1}}, \\
& \delta_{1}=\frac{\alpha_{1}\left(\alpha_{1}^{2} \beta_{1}-3 \gamma_{1}\right)}{2 \alpha_{1}+2 \beta_{1}+2 \gamma_{1}} .
\end{aligned}
$$

Therefore, the resulting one-wave solution reads as

$$
\Psi(x, y, z, t)=\frac{\sigma_{1}+\rho_{2}\left(2 \alpha_{1} \rho_{1}+\sigma_{1}\right) e^{\alpha_{1} x+\beta_{1} y+\gamma_{1} z-\left(\left(\alpha_{1}\left(\alpha_{1}^{2} \beta_{1}-3 \gamma_{1}\right)\right) /\left(2 \alpha_{1}+2 \beta_{1}+2 \gamma_{1}\right)\right) t}}{\rho_{1}+\rho_{2} e^{\alpha_{1} x+\beta_{1} y+\gamma_{1} z-\left(\left(\alpha_{1}\left(\alpha_{1}^{2} \beta_{1}-3 \gamma_{1}\right)\right) /\left(2 \alpha_{1}+2 \beta_{1}+2 \gamma_{1}\right)\right) t}} .
$$

By choosing the suitable values of parameters, the graphic representation of the periodic wave solution is presented in Figure 4 containing the 3D plot, the contour plot, the density plot, and the $2 \mathrm{D}$ plot when three spaces arise at spaces $x=-1$, $x=0$, and $x=1$.

4.2. Set II: Two-Wave Solutions. We commence with two-wave functions based on the statement in Step 2 in the previous section; we suppose that equation (2) has the rational function of two-wave solutions as shown in the following form:

$$
\begin{aligned}
\Psi(x, y, z, t)= & \frac{\Delta_{2}}{\Omega_{2}}, \\
\Omega_{2}= & 1+\sigma_{1} e^{\alpha_{1} x+\beta_{1} y+\gamma_{1} z-\delta_{1} t}+\sigma_{2} e^{\alpha_{2} x+\beta_{2} y+\gamma_{2} z-\delta_{2} t} \\
& +\sigma_{1} \sigma_{2} \sigma_{12} e^{\left(\alpha_{1}+\alpha_{2}\right) x+\left(\beta_{1}+\beta_{2}\right) y+\left(\gamma_{1}+\gamma_{2}\right) z-\left(\delta_{1}+\delta_{2}\right) t},
\end{aligned}
$$

$$
\begin{aligned}
\Delta_{2}= & \rho_{1} e^{\alpha_{1} x+\beta_{1} y+\gamma_{1} z-\delta_{1} t}+\rho_{2} e^{\alpha_{2} x+\beta_{2} y+\gamma_{2} z-\delta_{2} t} \\
& +\rho_{1} \rho_{2} \rho_{12} e^{\left(\alpha_{1}+\alpha_{2}\right) x+\left(\beta_{1}+\beta_{2}\right) y+\left(\gamma_{1}+\gamma_{2}\right) z-\left(\delta_{1}+\delta_{2}\right) t} .
\end{aligned}
$$

Plugging (27) along with (28) into equation (2), we get to the following case:

$$
\begin{gathered}
\sigma_{i}=\sigma_{i}, \\
\delta_{i}=\frac{\alpha_{i}^{3} \beta_{i}}{2 \alpha_{i}+2 \beta_{i}}, \\
\rho_{i}=2 \alpha_{i} \sigma_{i}, \\
i=1,2,
\end{gathered}
$$


(f1)

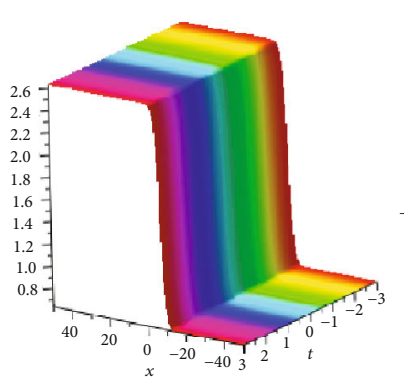

(f2)

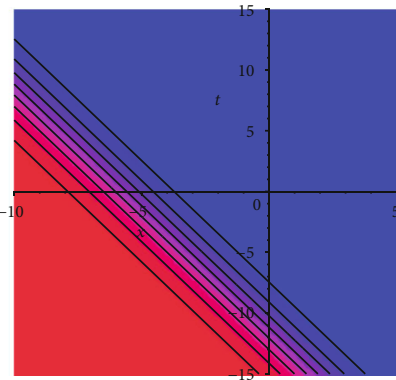

(f3)

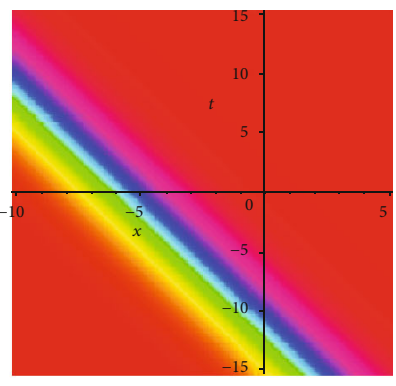

(f4)

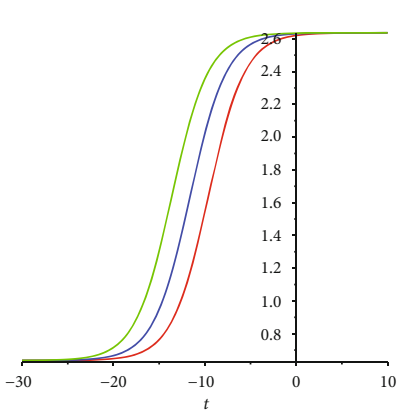

Figure 4: The one-wave solution (26) at $\alpha_{1}=1, \beta_{1}=1.5, \sigma_{1}=1.4, \rho_{1}=2.2, \rho_{2}=3, \gamma_{1}=2, y=1$, and $z=2$.

$$
\begin{aligned}
& \rho_{12}=\frac{\Sigma_{12}}{2 \alpha_{1} \alpha_{2} \Gamma_{12}}, \\
& \sigma_{12}=\frac{Y_{12}}{\Phi_{12}} .
\end{aligned}
$$$$
\Sigma_{12}=\alpha_{1} \alpha_{2}\left(\alpha_{1}^{2} \beta_{2}+2 \alpha_{1} \alpha_{2} \beta_{1}-2 \alpha_{1} \alpha_{2} \beta_{2}-\alpha_{2}^{2} \beta_{1}+3 \beta_{1}^{2} \beta_{2}\right.
$$$$
\left.-3 \beta_{1} \beta_{2}^{2}\right)\left(\alpha_{1}^{2}-\alpha_{2}^{2}\right)+\alpha_{2}^{2} \beta_{1}^{2}\left(\alpha_{1}+\alpha_{2}\right)\left(3 \alpha_{1}^{2}-3 \alpha_{1} \alpha_{2}+\alpha_{2}^{2}\right)
$$$$
+2 \alpha_{1} \alpha_{2} \beta_{1} \beta_{2}\left(\alpha_{1}+\alpha_{2}\right)\left(\alpha_{1}^{2}-3 \alpha_{1} \alpha_{2}+\alpha_{2}^{2}\right)
$$$$
+\alpha_{1}^{2} \beta_{2}^{2}\left(\alpha_{1}+\alpha_{2}\right)\left(\alpha_{1}^{2}-3 \alpha_{1} \alpha_{2}+3 \alpha_{2}^{2}\right)
$$$$
\Gamma_{12}=\alpha_{1} \alpha_{2}\left(\alpha_{1}+\alpha_{2}\right)\left(\alpha_{1}^{2} \beta_{2}+2 \alpha_{1} \alpha_{2} \beta_{1}+2 \alpha_{1} \alpha_{2} \beta_{2}+\alpha_{2}^{2} \beta_{1}\right.
$$$$
\left.+3 \beta_{1}^{2} \beta_{2}+3 \beta_{1} \beta_{2}^{2}\right)+\alpha_{2}^{2} \beta_{1}^{2}\left(3 \alpha_{1}^{2}+3 \alpha_{1} \alpha_{2}+\alpha_{2}^{2}\right)
$$$$
+2 \alpha_{1} \alpha_{2} \beta_{1} \beta_{2}\left(\alpha_{1}^{2}+3 \alpha_{1} \alpha_{2}+\alpha_{2}^{2}\right)
$$$$
+\alpha_{1}^{2} \beta_{2}^{2}\left(\alpha_{1}^{2}+3 \alpha_{1} \alpha_{2}+3 \alpha_{2}^{2}\right) \text {, }
$$$$
\mathrm{Y}_{12}=\alpha_{1} \alpha_{2}\left(\alpha_{1}-\alpha_{2}\right)\left(\alpha_{1}^{2} \beta_{2}+2 \alpha_{1} \alpha_{2} \beta_{1}-2 \alpha_{1} \alpha_{2} \beta_{2}-\alpha_{2}^{2} \beta_{1}\right.
$$$$
\left.+3 \beta_{1}^{2} \beta_{2}-3 \beta_{1} \beta_{2}^{2}\right)+\alpha_{2}^{2} \beta_{1}^{2}\left(3 \alpha_{1}^{2}-3 \alpha_{1} \alpha_{2}+\alpha_{2}^{2}\right)
$$$$
+2 \alpha_{1} \alpha_{2} \beta_{1} \beta_{2}\left(\alpha_{1}^{2}-3 \alpha_{1} \alpha_{2}+\alpha_{2}^{2}\right)
$$$$
+\alpha_{1}^{2} \beta_{2}^{2}\left(\alpha_{1}^{2}-3 \alpha_{1} \alpha_{2}+3 \alpha_{2}^{2}\right)
$$$$
\Phi_{12}=\alpha_{1} \alpha_{2}\left(\alpha_{1}+\alpha_{2}\right)\left(\alpha_{1}^{2} \beta_{2}+2 \alpha_{1} \alpha_{2} \beta_{1}+2 \alpha_{1} \alpha_{2} \beta_{2}+\alpha_{2}^{2} \beta_{1}\right.
$$$$
\left.+3 \beta_{1}^{2} \beta_{2}+3 \beta_{1} \beta_{2}^{2}\right)+\alpha_{2}^{2} \beta_{1}^{2}\left(3 \alpha_{1}^{2}+3 \alpha_{1} \alpha_{2}+\alpha_{2}^{2}\right)
$$$$
+2 \alpha_{1} \alpha_{2} \beta_{1} \beta_{2}\left(\alpha_{1}^{2}+3 \alpha_{1} \alpha_{2}+\alpha_{2}^{2}\right)
$$$$
+\alpha_{1}^{2} \beta_{2}^{2}\left(\alpha_{1}^{2}+3 \alpha_{1} \alpha_{2}+3 \alpha_{2}^{2}\right) \text {. }
$$

$$
\begin{gathered}
\sigma_{i}=\sigma_{i}, \\
\delta_{i}=\frac{\alpha_{i}^{3} \beta_{i}}{2 \alpha_{i}+2 \beta_{i}}, \\
\rho_{i}=2 \alpha_{i} \sigma_{i}, \\
i=1,2,3, \\
\rho_{12}=\frac{\Sigma_{12}}{2 \alpha_{1} \alpha_{2} \Gamma_{12}}, \\
\sigma_{12}=\frac{\mathrm{Y}_{12}}{\Phi_{12}} .
\end{gathered}
$$

in which $\Lambda_{i}=\alpha_{i} x+\beta_{i} y+\gamma_{i} z-\left(\alpha_{i}^{3} \beta_{i} /\left(2 \alpha_{i}+2 \beta_{i}\right)\right) t, i=1,2$. By selecting the suitable values of parameters, the graphic representation of the periodic wave solution is presented in Figure 5 including the $3 \mathrm{D}$ plot, the contour plot, the density three-wave functions based on the statement in Step 2 in the previous section; we suppose that equation (2) has the rational function of triple-wave solutions as shown in the following form:

$\Psi(x, y, z, t)=\frac{\Delta_{3}}{\Omega_{3}}$,

$$
\begin{aligned}
\Omega_{3}= & 1+\rho_{1} e^{\Lambda_{1}}+\rho_{2} e^{\Lambda_{2}}+\rho_{3} e^{\Lambda_{3}}+\rho_{1} \rho_{2} \rho_{12} e^{\Lambda_{1}+\Lambda_{2}} \\
& +\rho_{1} \rho_{3} \rho_{13} e^{\Lambda_{1}+\Lambda_{3}}+\rho_{2} \rho_{3} \rho_{23} e^{\Lambda_{2}+\Lambda_{3}} \\
& +\rho_{1} \rho_{2} \rho_{3} \rho_{12} \rho_{13} \rho_{23} e^{\Lambda_{1}+\Lambda_{2}+\Lambda_{3}}, \\
\Delta_{3}= & \sigma_{1} e^{\Lambda_{1}}+\sigma_{2} e^{\Lambda_{2}}+\sigma_{3} e^{\Lambda_{3}}+\sigma_{1} \sigma_{2} \sigma_{12} e^{\Lambda_{1}+\Lambda_{2}} \\
& +\sigma_{1} \sigma_{3} \sigma_{13} e^{\Lambda_{1}+\Lambda_{3}}+\sigma_{2} \sigma_{3} \sigma_{23} e^{\Lambda_{2}+\Lambda_{3}} \\
& +\sigma_{1} \sigma_{2} \sigma_{3} \sigma_{12} \sigma_{13} \sigma_{23} e^{\Lambda_{1}+\Lambda_{2}+\Lambda_{3}},
\end{aligned}
$$

in which $\Lambda_{i}=\alpha_{i} x+\beta_{i} y+\gamma_{i} x-\delta_{i} t, i=1,2,3$. Plugging (32) along with (33) into equation (2), we obtain the following case:

plot, and the 2D plot when three spaces arise at spaces 


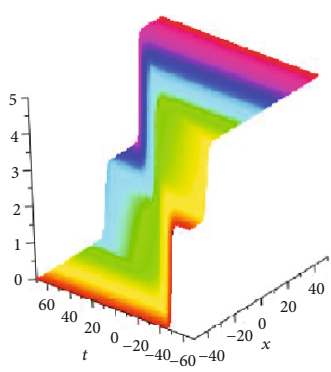

(f2)

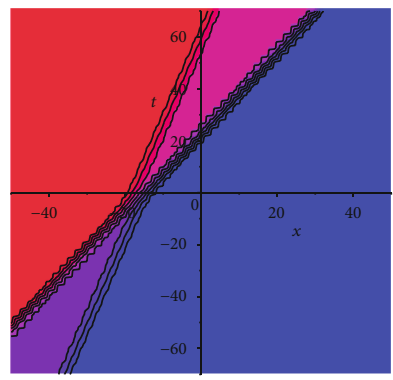

(f3)

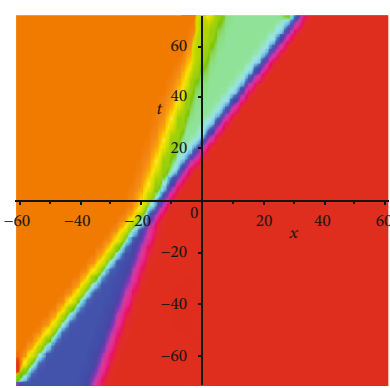

(f4)

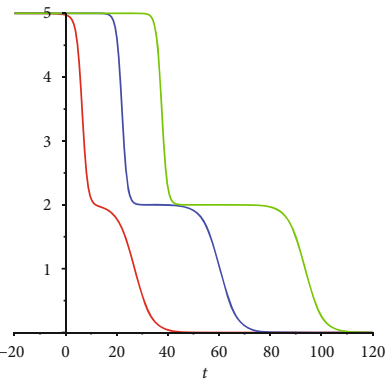

Figure 5: The two-wave solution (31) at $\alpha_{1}=1, \alpha_{2}=1.5, \beta_{1}=1.5, \beta_{2}=2, \sigma_{1}=1.1, \sigma_{2}=2, \gamma_{1}=1.5, \gamma_{2}=2, y=10$, and $z=2$.

$$
\begin{gathered}
\rho_{13}=\frac{\Sigma_{13}}{2 \alpha_{1} \alpha_{3} \Gamma_{13}}, \\
\sigma_{13}=\frac{Y_{13}}{\Phi_{13}}, \\
\rho_{23}=\frac{\Sigma_{23}}{2 \alpha_{2} \alpha_{3} \Gamma_{23}}, \\
\sigma_{23}=\frac{Y_{23}}{\Phi_{23}}, \\
\Sigma_{13}=\text { substitute }\left\{\alpha_{2}=\alpha_{3}, \beta_{2}=\beta_{3}\right\}, \text { at } \Sigma_{12}, \\
\Sigma_{23}=\text { substitute }\left\{\alpha_{1}=\alpha_{2}, \beta_{1}=\beta_{2}\right\}, \text { at } \Sigma_{13}, \\
\Gamma_{13}=\text { substitute }\left\{\alpha_{2}=\alpha_{3}, \beta_{2}=\beta_{3}\right\}, \text { at } \Gamma_{12}, \\
\Gamma_{23}=\text { substitute }\left\{\alpha_{1}=\alpha_{2}, \beta_{1}=\beta_{2}\right\}, \text { at } \Gamma_{13}, \\
Y_{13}=\text { substitute }\left\{\alpha_{2}=\alpha_{3}, \beta_{2}=\beta_{3}\right\}, \text { at } Y_{12}, \\
Y_{23}=\text { substitute }\left\{\alpha_{1}=\alpha_{2}, \beta_{1}=\beta_{2}\right\}, \text { at } Y_{13}, \\
\Phi_{13}=\text { substitute }\left\{\alpha_{2}=\alpha_{3}, \beta_{2}=\beta_{3}\right\}, \text { at } \Phi_{12}, \\
\Phi_{23}=\text { substitute }\left\{\alpha_{1}=\alpha_{2}, \beta_{1}=\beta_{2}\right\}, \text { at } \Phi_{13} .
\end{gathered}
$$

Therefore, the resulting three-wave solution reads as

$$
\Psi_{3}(x, y, z, t)=\frac{\Delta_{3}}{\Omega_{3}}
$$

in which $\Delta_{3}$ and $\Omega_{3}$ with their relations are given in (33) and (34). By selecting the suitable values of parameters, the graphic representation of the periodic wave solution is presented in Figure 6 containing the 3D plot, the contour plot, the density plot, and the $2 \mathrm{D}$ plot when three spaces arise at spaces $x=-10, x=0$, and $x=10$.

\section{Multiple Soliton Solutions for the $(2+1)$ eCBS Equation}

5.1. Set I: One-Wave Solution. We start with one-wave function based on the statement in Step 2 in the previous section; we suppose that equation (3) has the rational function of the one-wave solution as shown in the following form:

$$
\begin{aligned}
\Psi, y, t)=\frac{\Delta_{1}}{\Omega_{1}}, \quad \Omega_{1} & =\rho_{1}+\rho_{2} e^{\alpha_{1} x+\beta_{1} y-\lambda_{1} t}, \\
\Delta_{1} & =\sigma_{1}+\sigma_{2} e^{\alpha_{1} x+\beta_{1} y-\lambda_{1} t},
\end{aligned}
$$

in which $\sigma_{1}$ and $\sigma_{2}$ are unfound constants. Substituting (37) into equation (3), we get to the following case:

$$
\begin{aligned}
& \rho_{1}=\rho_{1}, \\
& \rho_{2}=\rho_{2}, \\
& \sigma_{1}=\sigma_{1}, \\
& \sigma_{2}=\frac{\rho_{2}\left(\alpha_{1} \rho_{1}+\sigma_{1}\right)}{\rho_{1}}, \\
& \lambda_{1}=\alpha_{1}^{3} \delta_{3}+\alpha_{1}^{2} \beta_{1}+\alpha_{1} \delta_{2}+\beta_{1} \delta_{1} .
\end{aligned}
$$

Therefore, the resulting one-wave solution reads as

$\Psi(x, y, t)=\frac{\sigma_{1}+\left(\rho_{2}\left(\alpha_{1} \rho_{1}+\sigma_{1}\right) / \rho_{1}\right) e^{\alpha_{1} x+\beta_{1} y-\left(\alpha_{1}^{3} \delta_{3}+\alpha_{1}^{2} \beta_{1}+\alpha_{1} \delta_{2}+\beta_{1} \delta_{1}\right) t}}{\rho_{1}+\rho_{2} e^{\alpha_{1} x+\beta_{1} y-\left(\alpha_{1}^{3} \delta_{3}+\alpha_{1}^{2} \beta_{1}+\alpha_{1} \delta_{2}+\beta_{1} \delta_{1}\right) t}}$.

By selecting the suitable values of parameters, the graphic representation of the periodic wave solution is presented in Figure 7 containing the $3 \mathrm{D}$ plot, the contour plot, the density plot, and the 2D plot when three spaces arise at spaces $x=-1$, $x=0$, and $x=1$.

5.2. Set II: Two-Wave Solutions. We begin with two-wave functions based on the statement in Step 2 in the previous section; we suppose that equation (3) has the rational function of two-wave solutions as shown in the following form:

$$
\Psi(x, y, z, t)=\frac{\Delta_{2}}{\Omega_{2}},
$$




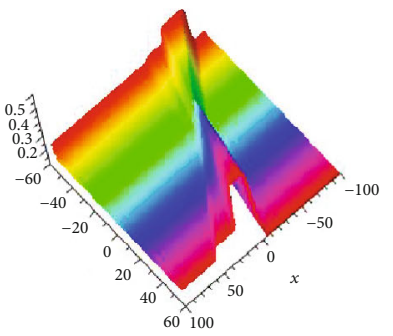

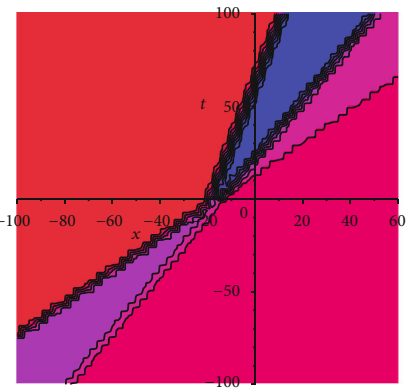

(f3)

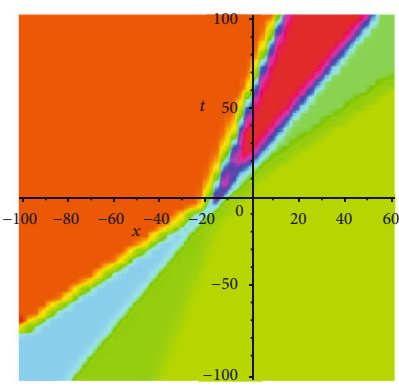

(f4)

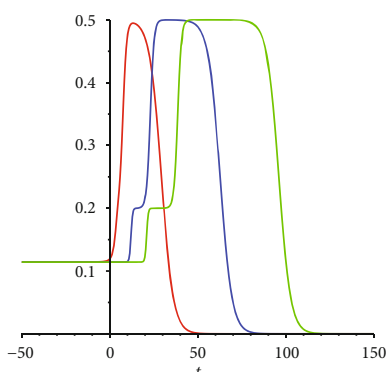

Figure 6: The three-wave solution (36) at $\alpha_{1}=1, \alpha_{2}=1.5, \alpha_{3}=2, \sigma_{1}=1.1, \sigma_{2}=2, \sigma_{3}=2.7, \beta_{1}=2.5, \beta_{2}=2, \beta_{3}=2.6, \gamma_{1}=1.5, \gamma_{2}=2, \gamma_{3}=3$, $y=10$, and $z=2$.

(f1)

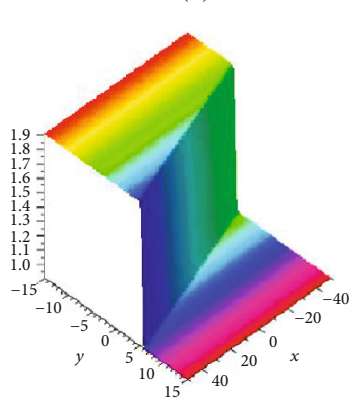

(f2)

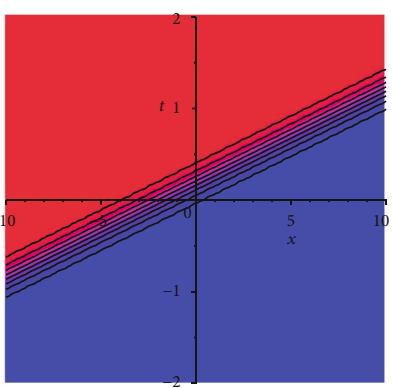

(f3)

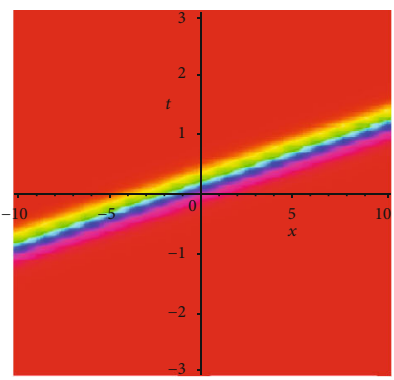

(f4)

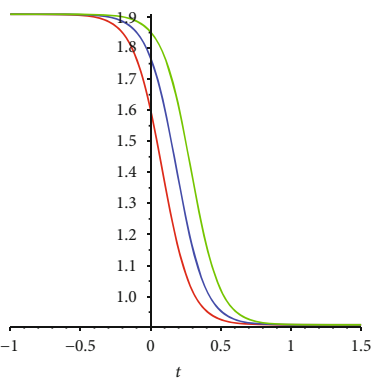

FIgURE 7: The one-wave solution (39) at $\alpha_{1}=1, \sigma_{1}=2, \rho_{1}=2.2, \rho_{2}=3, \rho_{1}=2, \beta_{1}=1.5, \delta_{1}=1.5, \delta_{2}=2.5, \delta_{3}=0.5$, and $y=1$.

$$
\begin{aligned}
\Omega_{2}= & 1+\sigma_{1} e^{\alpha_{1} x+\beta_{1} y-\lambda_{1} t}+\sigma_{2} e^{\alpha_{2} x+\beta_{2} y-\lambda_{2} t} \\
& +\sigma_{1} \sigma_{2} \sigma_{12} e^{\left(\alpha_{1}+\alpha_{2}\right) x+\left(\beta_{1}+\beta_{2}\right) y+\left(\gamma_{1}-\left(\lambda_{1}+\lambda_{2}\right) t\right.}, \\
\Delta_{2}= & \rho_{1} e^{\alpha_{1} x+\beta_{1} y-\lambda_{1} t}+\rho_{2} e^{\alpha_{2} x+\beta_{2} y-\lambda_{2} t} \\
& +\rho_{1} \rho_{2} \rho_{12} e^{\left(\alpha_{1}+\alpha_{2}\right) x+\left(\beta_{1}+\beta_{2}\right) y-\left(\lambda_{1}+\lambda_{2}\right) t} .
\end{aligned}
$$

Inserting (40) along with (41) into equation (3), we obtain the following case:

$$
\begin{aligned}
& \sigma_{i}=\sigma_{i}, \\
& \lambda_{i}=\alpha_{i}^{3} \delta_{3}+\alpha_{i}^{2} \beta_{i}+\alpha_{i} \delta_{2}+\beta_{i} \delta_{1}, \\
& \rho_{i}=2 \alpha_{i} \sigma_{i}, \\
& \quad i=1,2, \\
& \rho_{12}=\frac{3 \alpha_{1} \alpha_{2} \delta_{3}\left(\alpha_{1}-\alpha_{2}\right)^{2}+\left(\alpha_{1}-\alpha_{2}\right)\left(\alpha_{1}^{2} \beta_{2}+2 \alpha_{1} \alpha_{2} \beta_{1}-2 \alpha_{1} \alpha_{2} \beta_{2}-\alpha_{2}^{2} \beta_{1}\right)}{\alpha_{2} \alpha_{1}\left(3 \alpha_{1} \alpha_{2} \delta_{3}\left(\alpha_{1}+\alpha_{2}\right)+2 \alpha_{1} \alpha_{2}\left(\beta_{1}+\beta_{2}\right)+\alpha_{1}^{2} \beta_{2}+\alpha_{2}^{2} \beta_{1}\right)} . \\
& \sigma_{12}=\frac{3 \alpha_{1} \alpha_{2} \delta_{3}\left(\alpha_{1}-\alpha_{2}\right)^{2}+\left(\alpha_{1}-\alpha_{2}\right)\left(\alpha_{1}^{2} \beta_{2}+2 \alpha_{1} \alpha_{2} \beta_{1}-2 \alpha_{1} \alpha_{2} \beta_{2}-\alpha_{2}^{2} \beta_{1}\right)}{3 \alpha_{1} \alpha_{2} \delta_{3}\left(\alpha_{1}+\alpha_{2}\right)^{2}+\left(\alpha_{1}+\alpha_{2}\right)\left(\alpha_{1}^{2} \beta_{2}+2 \alpha_{1} \alpha_{2} \beta_{1}+2 \alpha_{1} \alpha_{2} \beta_{2}+\alpha_{2}^{2} \beta_{1}\right)} .
\end{aligned}
$$

Therefore, the resulting two-wave solution reads as

$$
\Psi_{2}(x, y, t)=\frac{\sigma_{1} e^{\Lambda_{1}}+\sigma_{2} e^{\Lambda_{2}}+\sigma_{1} \sigma_{2} \sigma_{12} e^{\Lambda_{1}+\Lambda_{2}}}{\rho_{1} e^{\Lambda_{1}}+\rho_{2} e^{\Lambda_{2}}+4 \alpha_{1} \alpha_{2} \sigma_{1} \sigma_{2} \rho_{12} e^{\Lambda_{1}+\Lambda_{2}}},
$$

in which $\Lambda_{i}=\alpha_{i} x+\beta_{i} y-\left(\alpha_{i}^{3} \delta_{3}+\alpha_{i}^{2} \beta_{i}+\alpha_{i} \delta_{2}+\beta_{i} \delta_{1}\right) t, i=$ 1,2 . By choosing the suitable values of parameters, the graphic representation of the periodic wave solution is presented in Figure 8 including the 3D plot, the contour plot, 
(f1)

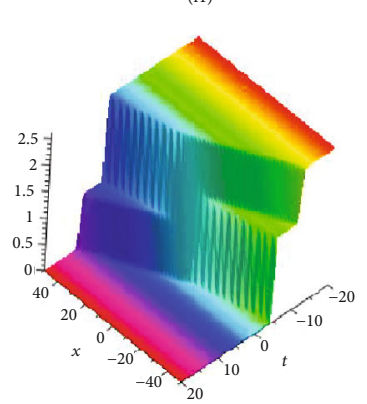

(f2)

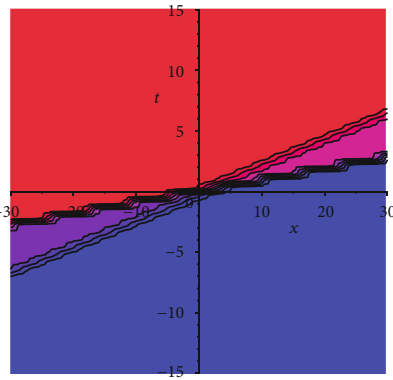

(f3)

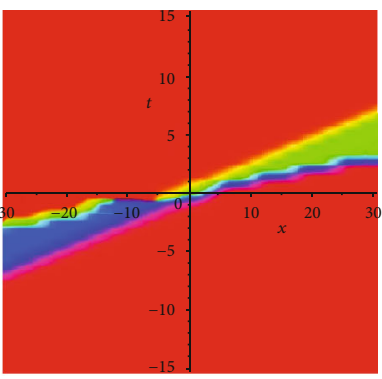

(f4)

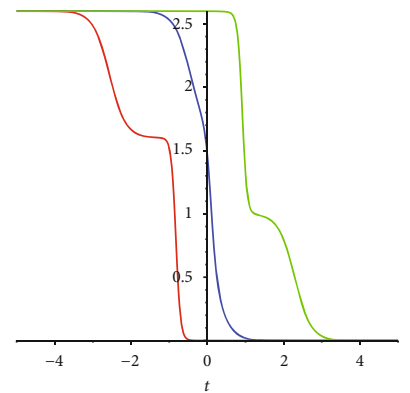

Figure 8: The two-wave solution (44) at $\alpha_{1}=1, \alpha_{2}=1.6, \sigma_{2}=1.5, \rho=1.5, \rho_{1}=2, \rho_{2}=3, \beta_{1}=2.5, \beta_{2}=3.4, \gamma_{1}=0.75, \gamma_{2}=1.5, a(t)=\sin (t)$, $b(t)=\cos (t), y=1$, and $z=2$.

the density plot, and the $2 \mathrm{D}$ plot when three spaces arise at spaces $x=-1, x=0$, and $x=1$.

5.3. Set III: Triple-Wave Solutions. We commence with threewave functions based on the statement in Step 2 in the previous section; we suppose that equation (3) has the rational function of triple-wave solutions as shown in the following form:

$$
\Psi(x, y, z, t)=\frac{\Delta_{3}}{\Omega_{3}},
$$

$$
\begin{aligned}
\Omega_{3}= & +\rho_{1} e^{\Lambda_{1}}+\rho_{2} e^{\Lambda_{2}}+\rho_{3} e^{\Lambda_{3}}+\rho_{1} \rho_{2} \rho_{12} e^{\Lambda_{1}+\Lambda_{2}} \\
& +\rho_{1} \rho_{3} \rho_{13} e^{\Lambda_{1}+\Lambda_{3}}+\rho_{2} \rho_{3} \rho_{23} e^{\Lambda_{2}+\Lambda_{3}} \\
& +\rho_{1} \rho_{2} \rho_{3} \rho_{12} \rho_{13} \rho_{23} e^{\Lambda_{1}+\Lambda_{2}+\Lambda_{3}}, \\
\Delta_{3}= & \sigma_{1} e^{\Lambda_{1}}+\sigma_{2} e^{\Lambda_{2}}+\sigma_{3} e^{\Lambda_{3}}+\sigma_{1} \sigma_{2} \sigma_{12} e^{\Lambda_{1}+\Lambda_{2}} \\
& +\sigma_{1} \sigma_{3} \sigma_{13} e^{\Lambda_{1}+\Lambda_{3}}+\sigma_{2} \sigma_{3} \sigma_{23} e^{\Lambda_{2}+\Lambda_{3}} \\
& +\sigma_{1} \sigma_{2} \sigma_{3} \sigma_{12} \sigma_{13} \sigma_{23} e^{\Lambda_{1}+\Lambda_{2}+\Lambda_{3}},
\end{aligned}
$$

in which $\Lambda_{i}=\alpha_{i} x+\beta_{i} y-\lambda_{i} t, i=1,2,3$. Plugging (45) along with (46) into equation (3), we obtain the following case:

$$
\begin{aligned}
& \sigma_{i}=\sigma_{i}, \\
& \lambda_{i}=\alpha_{i}^{3} \delta_{3}+\alpha_{i}^{2} \beta_{i}+\alpha_{i} \delta_{2}+\beta_{i} \delta_{1}, \\
& \rho_{i}=2 \alpha_{i} \sigma_{i}, \\
& i=1,2,3, \\
& \rho_{12}=\frac{3 \alpha_{1} \alpha_{2} \delta_{3}\left(\alpha_{1}-\alpha_{2}\right)^{2}+\left(\alpha_{1}-\alpha_{2}\right)\left(\alpha_{1}^{2} \beta_{2}+2 \alpha_{1} \alpha_{2} \beta_{1}-2 \alpha_{1} \alpha_{2} \beta_{2}-\alpha_{2}^{2} \beta_{1}\right)}{\alpha_{2} \alpha_{1}\left(3 \alpha_{1} \alpha_{2} \delta_{3}\left(\alpha_{1}+\alpha_{2}\right)+2 \alpha_{1} \alpha_{2}\left(\beta_{1}+\beta_{2}\right)+\alpha_{1}^{2} \beta_{2}+\alpha_{2}^{2} \beta_{1}\right)}, \\
& \rho_{12}=\frac{3 \alpha_{1} \alpha_{2} \delta_{3}\left(\alpha_{1}-\alpha_{2}\right)^{2}+\left(\alpha_{1}-\alpha_{2}\right)\left(\alpha_{1}^{2} \beta_{2}+2 \alpha_{1} \alpha_{2} \beta_{1}-2 \alpha_{1} \alpha_{2} \beta_{2}-\alpha_{2}^{2} \beta_{1}\right)}{3 \alpha_{1} \alpha_{2} \delta_{3}\left(\alpha_{1}+\alpha_{2}\right)^{2}+\left(\alpha_{1}+\alpha_{2}\right)\left(\alpha_{1}^{2} \beta_{2}+2 \alpha_{1} \alpha_{2} \beta_{1}+2 \alpha_{1} \alpha_{2} \beta_{2}+\alpha_{2}^{2} \beta_{1}\right)}, \\
& \sigma_{13}=\text { substitute }\left\{\alpha_{2}=\alpha_{3}, \beta_{2}=\beta_{3}\right\}, \text { at } \rho_{12}, \\
& \rho_{23}=\text { substitute }\left\{\alpha_{2}=\alpha_{3}, \beta_{2}=\beta_{3}\right\}, \text { at } \sigma_{12}, \\
& \sigma_{23}=\text { substitute }\left\{\alpha_{1}=\alpha_{2}, \beta_{1}=\beta_{2}, \beta_{1}=\beta_{2}\right\}, \text { at } \rho_{13},
\end{aligned}
$$

Therefore, the resulting three-wave solution reads as

$$
\Psi_{3}(x, y, z, t)=\frac{\Delta_{3}}{\Omega_{3}} .
$$

In (48), $\Delta_{3}$ and $\Omega_{3}$ with their relations are given in (46) and (47). By selecting the suitable values of parameters, the graphic representation of the periodic wave solution is presented in Figure 9 containing the 3D plot, the contour plot, the density plot, and the $2 \mathrm{D}$ plot when three spaces arise at spaces $x=-1, x=0$, and $x=1$. 


\section{Multiple Soliton Solutions for the Generalized $(2+1)$ BK Equation}

6.1. Set I: One-Wave Solution. We start with one-wave function based on the statement in Step 2 in the previous section; we suppose that equation (4) has the rational function of the one-wave solution as shown in the following form:

$$
\begin{aligned}
\Psi(x, y, t)=\frac{\Delta_{1}}{\Omega_{1}}, \quad \Omega_{1} & =\rho_{1}+\rho_{2} e^{\alpha_{1} x+\beta_{1} y-\lambda_{1} t}, \\
\Delta_{1} & =\sigma_{1}+\sigma_{2} e^{\alpha_{1} x+\beta_{1} y-\lambda_{1} t},
\end{aligned}
$$

in which $\sigma_{1}$ and $\sigma_{2}$ are unfound constants. Putting (49) into equation (4), we get to the following case:

$$
\begin{aligned}
& \rho_{1}=\rho_{1}, \\
& \rho_{2}=\rho_{2}, \\
& \sigma_{1}=\sigma_{1}, \\
& \sigma_{2}=\frac{\rho_{2}\left(2 \alpha_{1} \rho_{1}+\sigma_{1}\right)}{\rho_{1}}, \\
& \lambda_{1}=\frac{\alpha \alpha_{1}^{4}+\beta \alpha_{1}^{3} \beta_{1}+\alpha_{1}^{2} \delta_{1}+\alpha_{1} \beta_{1} \delta_{2}+\beta_{1}^{2} \delta_{3}}{\alpha_{1}} .
\end{aligned}
$$

Therefore, the resulting one-wave solution reads as

$$
\Psi(x, y, t)=\frac{\sigma_{1}+\left(\rho_{2}\left(2 \alpha_{1} \rho_{1}+\sigma_{1}\right) / \rho_{1}\right) e^{\alpha_{1} x+\beta_{1} y-\left(\alpha \alpha_{1}^{4}+\beta \alpha_{1}^{3} \beta_{1}+\alpha_{1}^{2} \delta_{1}+\alpha_{1} \beta_{1} \delta_{2}+\beta_{1}^{2} \delta_{3} / \alpha_{1}\right) t}}{\rho_{1}+\rho_{2} e^{\alpha_{1} x+\beta_{1} y-\left(\alpha \alpha_{1}^{4}+\beta \alpha_{1}^{3} \beta_{1}+\alpha_{1}^{2} \delta_{1}+\alpha_{1} \beta_{1} \delta_{2}+\beta_{1}^{2} \delta_{3} / \alpha_{1}\right) t}} .
$$

By selecting the suitable values of parameters, the graphic representation of the periodic wave solution is presented in Figure 10 containing the 3D plot, the contour plot, the density plot, and the $2 \mathrm{D}$ plot when three spaces arise at spaces $x=-10, x=0$, and $x=10$.

6.2. Set II: Two-Wave Solutions. We begin with two-wave functions based on the statement in Step 2 in the previous section; we suppose that equation (4) has the rational function of two-wave solutions as shown in the following form:

$$
\begin{aligned}
\Psi(x, y, z, t)= & \frac{\Delta_{2}}{\Omega_{2}} \\
\Omega_{2}= & 1+\sigma_{1} e^{\alpha_{1} x+\beta_{1} y-\lambda_{1} t}+\sigma_{2} e^{\alpha_{2} x+\beta_{2} y-\lambda_{2} t} \\
& +\sigma_{1} \sigma_{2} \sigma_{12} e^{\left(\alpha_{1}+\alpha_{2}\right) x+\left(\beta_{1}+\beta_{2}\right) y+\left(\gamma_{1}-\left(\lambda_{1}+\lambda_{2}\right) t\right.}, \\
\Delta_{2}= & \rho_{1} e^{\alpha_{1} x+\beta_{1} y-\lambda_{1} t}+\rho_{2} e^{\alpha_{2} x+\beta_{2} y-\lambda_{2} t} \\
& +\rho_{1} \rho_{2} \rho_{12} e^{\left(\alpha_{1}+\alpha_{2}\right) x+\left(\beta_{1}+\beta_{2}\right) y-\left(\lambda_{1}+\lambda_{2}\right) t} .
\end{aligned}
$$

Inserting (52) along with (53) into equation (4), we obtain the following case:

$$
\begin{aligned}
& \sigma_{i}=\sigma_{i}, \\
& \lambda_{i}=\frac{\alpha \alpha_{i}^{4}+\beta \alpha_{i}^{3} \beta_{i}+\alpha_{i}^{2} \delta_{1}+\alpha_{i} \beta_{i} \delta_{2}+\beta_{i}^{2} \delta_{3}}{\alpha_{i}}, \\
& \rho_{i}=2 \alpha_{i} \sigma_{i}, \quad i=1,2 \\
& \rho_{12}= \\
& \sigma_{12}=\frac{3 \alpha \alpha_{1}^{2} \alpha_{2}^{2}\left(\alpha_{2}+\alpha_{1}\right)\left(\alpha_{1}-\alpha_{2}\right)^{2}+\left(\alpha_{2}+\alpha_{1}\right)\left(\beta \alpha_{1} \alpha_{2}\left(\alpha_{1}-\alpha_{2}\right)\left(\alpha_{1}^{2} \beta_{2}+2 \alpha_{1} \alpha_{2} \beta_{1}-2 \alpha_{1} \alpha_{2} \beta_{2}-\alpha_{2}^{2} \beta_{1}\right)-\delta_{3}\left(\alpha_{1} \beta_{2}-\alpha_{2} \beta_{1}\right)^{2}\right)}{2 \alpha_{1} \alpha_{2}\left(\alpha_{1} \alpha_{2}\left(\alpha_{2}+\alpha_{1}\right)\left(3 \alpha \alpha_{1} \alpha_{2}\left(\alpha_{2}+\alpha_{1}\right)+\beta\left(\alpha_{1}^{2} \beta_{2}+2 \alpha_{1} \alpha_{2} \beta_{1}+2 \alpha_{1} \alpha_{2} \beta_{2}+\alpha_{2}^{2} \beta_{1}\right)\right)-\delta_{3}\left(\alpha_{1} \beta_{2}-\alpha_{2} \beta_{1}\right)^{2}\right)} \\
& \alpha_{1} \alpha_{2}\left(\alpha_{2}+\alpha_{1}\right)\left(3 \alpha \alpha_{1} \alpha_{2}\left(\alpha_{2}+\alpha_{1}\right)+\beta\left(\alpha_{1}^{2} \beta_{2}+2 \alpha_{1} \alpha_{2} \beta_{1}+2 \alpha_{1} \alpha_{2} \beta_{2}+\alpha_{2}^{2} \beta_{1}\right)\right)-\delta_{3}\left(\alpha_{1} \beta_{2}-\alpha_{2} \beta_{1}\right)^{2}
\end{aligned}
$$



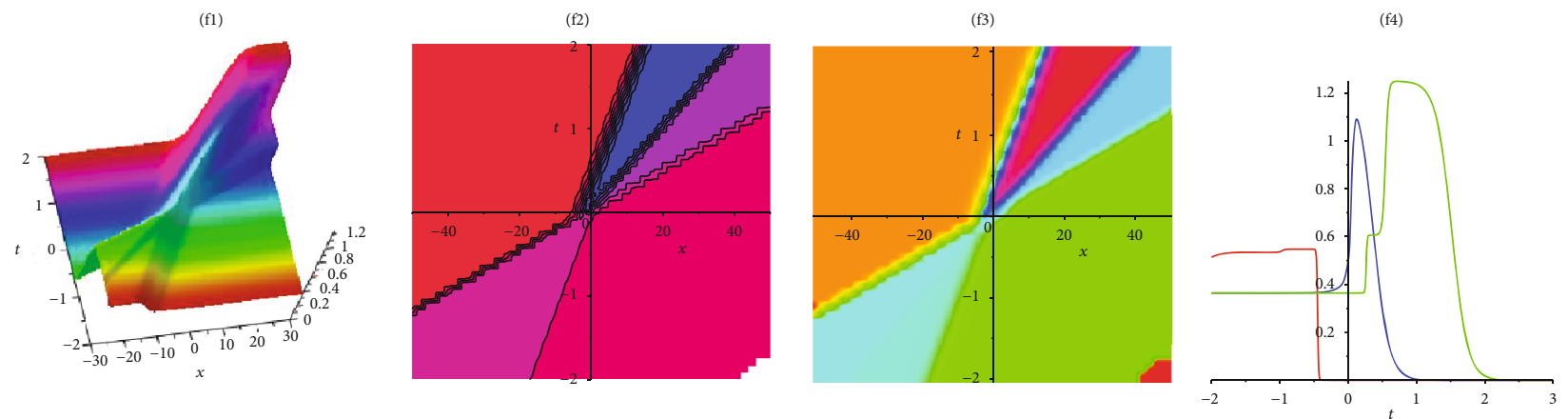

FIGURE 9: The three-wave solution (48) at $\alpha_{1}=1, \alpha_{2}=2.3, \alpha_{3}=3.6, \rho_{1}=2, \rho_{2}=2.3, \rho_{3}=4.2, \sigma_{1}=0.5, \sigma_{2}=1.6, \sigma_{3}=2.3, \delta_{1}=1, \delta_{2}=0.5$, $\delta_{3}=2.2, \beta_{1}=1.5, \beta_{2}=3.3$, and $\beta_{3}=5.4$.

Therefore, the resulting two-wave solution reads as

$$
\Psi_{2}(x, y, t)=\frac{\sigma_{1} e^{\Lambda_{1}}+\sigma_{2} e^{\Lambda_{2}}+\sigma_{1} \sigma_{2} \sigma_{12} e^{\Lambda_{1}+\Lambda_{2}}}{\rho_{1} e^{\Lambda_{1}}+\rho_{2} e^{\Lambda_{2}}+4 \alpha_{1} \alpha_{2} \sigma_{1} \sigma_{2} \rho_{12} e^{\Lambda_{1}+\Lambda_{2}}},
$$

in which $\Lambda_{i}=\alpha_{i} x+\beta_{i} y-\left(\alpha \alpha_{i}^{4}+\beta \alpha_{i}^{3} \beta_{i}+\alpha_{i}^{2} \delta_{1}+\alpha_{i} \beta_{i} \delta_{2}+\beta_{i}^{2}\right.$ $\left.\delta_{3} / \alpha_{i}\right) t, i=1,2$. By choosing suitable values of parameters, the analytical treatment of the periodic wave solution is presented in Figure 11 including the 3D plot, the contour plot, the density plot, and the $2 \mathrm{D}$ plot when three spaces arise at spaces $x=-10, x=0$, and $x=10$.

6.3. Set III: Triple-Wave Solutions. We commence with three-wave functions based on the statement in Step 2 in the previous section; we suppose that equation (4) has the rational function of triple-wave solutions as shown in the following form:

$$
\Psi(x, y, z, t)=\frac{\Delta_{3}}{\Omega_{3}},
$$

$$
\begin{aligned}
\Omega_{3}= & +\rho_{1} e^{\Lambda_{1}}+\rho_{2} e^{\Lambda_{2}}+\rho_{3} e^{\Lambda_{3}}+\rho_{1} \rho_{2} \rho_{12} e^{\Lambda_{1}+\Lambda_{2}} \\
& +\rho_{1} \rho_{3} \rho_{13} e^{\Lambda_{1}+\Lambda_{3}}+\rho_{2} \rho_{3} \rho_{23} e^{\Lambda_{2}+\Lambda_{3}} \\
& +\rho_{1} \rho_{2} \rho_{3} \rho_{12} \rho_{13} \rho_{23} e^{\Lambda_{1}+\Lambda_{2}+\Lambda_{3}}, \\
\Delta_{3}= & \sigma_{1} e^{\Lambda_{1}}+\sigma_{2} e^{\Lambda_{2}}+\sigma_{3} e^{\Lambda_{3}}+\sigma_{1} \sigma_{2} \sigma_{12} e^{\Lambda_{1}+\Lambda_{2}} \\
& +\sigma_{1} \sigma_{3} \sigma_{13} e^{\Lambda_{1}+\Lambda_{3}}+\sigma_{2} \sigma_{3} \sigma_{23} e^{\Lambda_{2}+\Lambda_{3}} \\
& +\sigma_{1} \sigma_{2} \sigma_{3} \sigma_{12} \sigma_{13} \sigma_{23} e^{\Lambda_{1}+\Lambda_{2}+\Lambda_{3}},
\end{aligned}
$$

in which $\Lambda_{i}=\alpha_{i} x+\beta_{i} y-\lambda_{i} t, i=1,2,3$. Plugging (56) along with (57) into equation (4), we obtain the following case:

$$
\begin{aligned}
& \sigma_{i}=\sigma_{i} \\
& \lambda_{i}=\frac{\alpha \alpha_{i}^{4}+\beta \alpha_{i}^{3} \beta_{i}+\alpha_{i}^{2} \delta_{1}+\alpha_{i} \beta_{i} \delta_{2}+\beta_{i}^{2} \delta_{3}}{\alpha_{i}}, \\
& \rho_{i}=2 \alpha_{i} \sigma_{i}, \\
& i=1,2,3 \text {, } \\
& \rho_{12}=\frac{3 \alpha \alpha_{1}^{2} \alpha_{2}^{2}\left(\alpha_{2}+\alpha_{1}\right)\left(\alpha_{1}-\alpha_{2}\right)^{2}+\left(\alpha_{2}+\alpha_{1}\right)\left(\beta \alpha_{1} \alpha_{2}\left(\alpha_{1}-\alpha_{2}\right)\left(\alpha_{1}^{2} \beta_{2}+2 \alpha_{1} \alpha_{2} \beta_{1}-2 \alpha_{1} \alpha_{2} \beta_{2}-\alpha_{2}^{2} \beta_{1}\right)-\delta_{3}\left(\alpha_{1} \beta_{2}-\alpha_{2} \beta_{1}\right)^{2}\right.}{2 \alpha_{1} \alpha_{2}\left(\alpha_{1} \alpha_{2}\right)\left(\alpha_{2}+\alpha_{1}\left(3 \alpha \alpha_{1} \alpha_{2}\left(\alpha_{2}+\alpha_{1}\right)+\beta\left(\alpha_{1}^{2} \beta_{2}+2 \alpha_{1} \alpha_{2} \beta_{1}+2 \alpha_{1} \alpha_{2} \beta_{2}+\alpha_{2}^{2} \beta_{1}\right)\right)-\delta_{3}\left(\alpha_{1} \beta_{2}-\alpha_{2} \beta_{1}\right)^{2}\right)}, \\
& \sigma_{12}=\frac{\alpha_{1} \alpha_{2}\left(-\alpha_{2}+\alpha_{1}\right)\left(3 \alpha \alpha_{1} \alpha_{2}\left(-\alpha_{2}+\alpha_{1}\right)+\beta\left(\alpha_{1}^{2} \beta_{2}+2 \alpha_{1} \alpha_{2} \beta_{1}-2 \alpha_{1} \alpha_{2} \beta_{2}-\alpha_{2}^{2} \beta_{1}\right)\right)-\delta_{3}\left(\alpha_{1} \beta_{2}-\alpha_{2} \beta_{1}\right)^{2}}{\alpha_{1} \alpha_{2}\left(\alpha_{2}+\alpha_{1}\right)\left(3 \alpha \alpha_{1} \alpha_{2}\left(\alpha_{2}+\alpha_{1}\right)+\beta\left(\alpha_{1}^{2} \beta_{2}+2 \alpha_{1} \alpha_{2} \beta_{1}+2 \alpha_{1} \alpha_{2} \beta_{2}+\alpha_{2}^{2} \beta_{1}\right)\right)-\delta_{3}\left(\alpha_{1} \beta_{2}-\alpha_{2} \beta_{1}\right)^{2}}, \\
& \rho_{13}=\text { substitute }\left\{\alpha_{2}=\alpha_{3}, \beta_{2}=\beta_{3}\right\} \text {, at } \rho_{12} \text {, } \\
& \sigma_{13}=\text { substitute }\left\{\alpha_{2}=\alpha_{3}, \beta_{2}=\beta_{3}\right\} \text {, at } \sigma_{12} \text {, } \\
& \rho_{23}=\text { substitute }\left\{\alpha_{1}=\alpha_{2}, \beta_{1}=\beta_{2}\right\} \text {, at } \rho_{13} \text {, } \\
& \sigma_{23}=\text { substitute }\left\{\alpha_{1}=\alpha_{2}, \beta_{1}=\beta_{2}\right\} \text {, at } \sigma_{13} \text {. }
\end{aligned}
$$




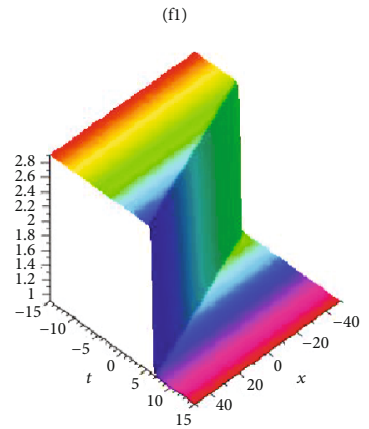

(f2)

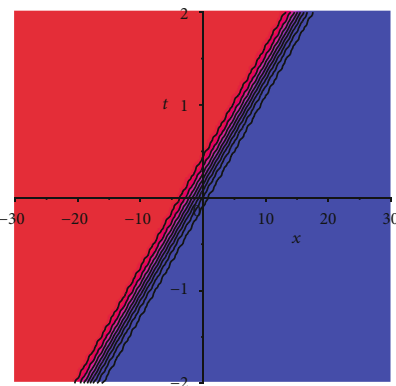

(f3)

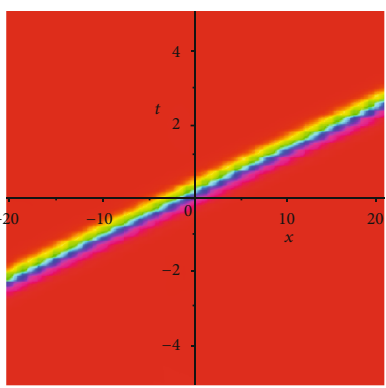

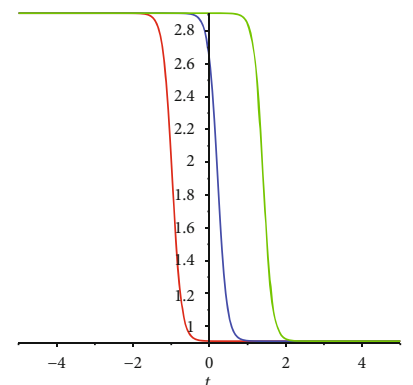

Figure 10: The one-wave solution (51) at $\alpha=0.5, \beta=0.1, \alpha_{1}=1, \beta_{1}=1.5, \sigma_{1}=2, \mathrm{rho}_{1}=2.2, \mathrm{rho}_{2}=3, \delta_{1}=1, \delta_{2}=1.5, \delta_{3}=2, \delta_{1}=1, \delta_{2}=$ $1.5, \delta_{3}=2$, and $y=1$.

(f1)

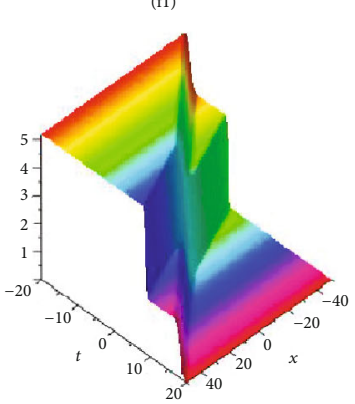

(f2)

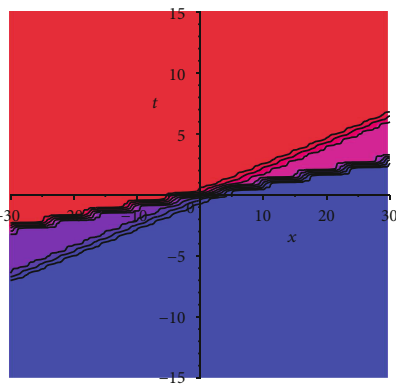

(f3)

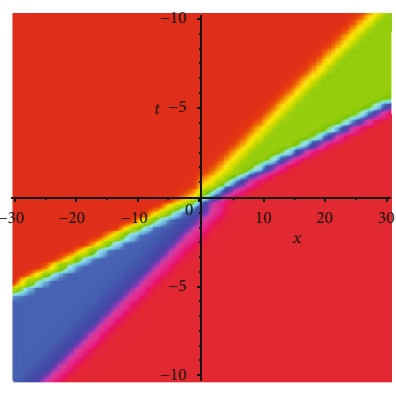

(f4)

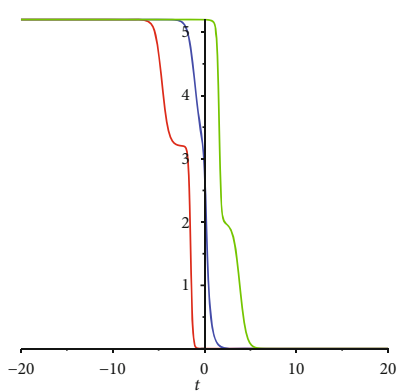

Figure 11: The two-wave solution (55) at $\alpha=0.5, \beta=0.1, \alpha_{1}=1, \alpha_{2}=1.6, \mathrm{rho}_{1}=2, \mathrm{rho}_{2}=2.3, \sigma_{1}=1.3, \sigma_{2}=2, \beta_{1}=0.5, \beta_{2}=1.5, \delta_{1}=0.1$, $\delta_{2}=0.5, \delta_{3}=0.2$, and $y=1$.

$(\mathrm{fl})$

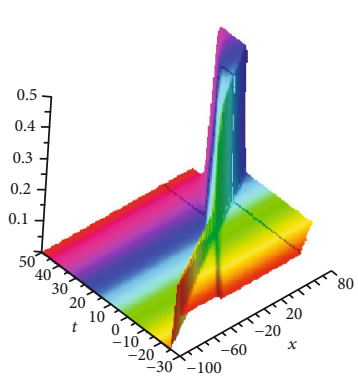

(f2)

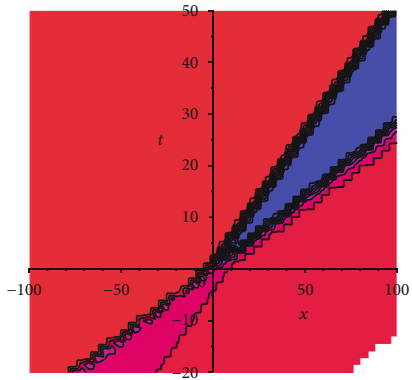

(f3)

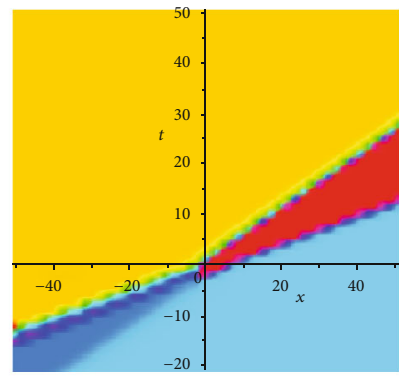

(f4)

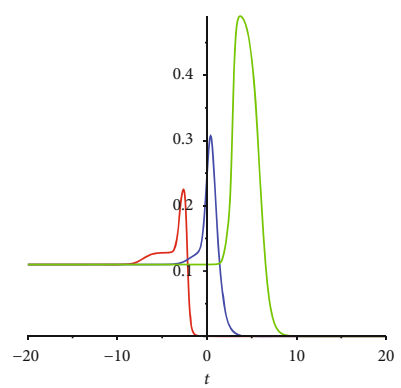

FIGURE 12: The three-wave solution (59) at alpha $=0.5$, beta $=0.1, \alpha_{1}=1, \alpha_{2}=2.3, \alpha_{3}=3.6, \rho_{1}=2, \rho_{2}=2.3, \rho_{3}=4.2, \sigma_{1}=0.5, \sigma_{2}=1.6, \sigma_{3}=$ $2.3, \delta_{1}=1, \delta_{2}=0.5, \delta_{3}=2.2, \beta_{1}=1.5, \beta_{2}=3.3$, and $\beta_{3}=5.4$.

Therefore, the resulting three-wave solution reads as

$$
\Psi_{3}(x, y, z, t)=\frac{\Delta_{3}}{\Omega_{3}}
$$

in which $\Delta_{3}$ and $\Omega_{3}$ with their relations are given in (57) and (58). By selecting the suitable values of parameters, the graphic representation of the periodic wave solution is presented in Figure 12 containing the 3D plot, the contour plot, the density plot, and the $2 \mathrm{D}$ plot when three spaces arise at spaces $x=-10, x=0$, and $x=10$.

\section{Multiple Soliton Solutions for the $(2+1)$ VC DJKM Equation}

7.1. Set I: One-Wave Solution. We start with one-wave function based on the statement in Step 2 in the previous section; we suppose that equation (6) has the rational function of one-wave solutions as shown in the following form:

$$
\begin{aligned}
& \Psi(x, y, t)=\frac{\Delta_{1}}{\Omega_{1}}, \quad \Omega_{1}=\rho_{1}+\rho_{2} e^{\alpha_{1} x+\beta_{1} y-\lambda_{1} t}, \\
& \Delta_{1}=\sigma_{1}+\sigma_{2} e^{\alpha_{1} x+\beta_{1} y-\lambda_{1} t},
\end{aligned}
$$


(f1)

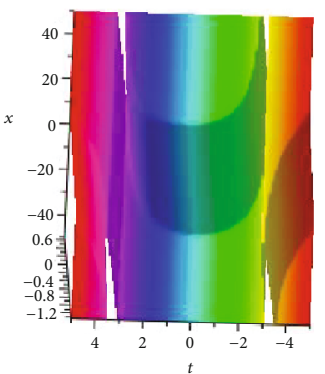

(f2)

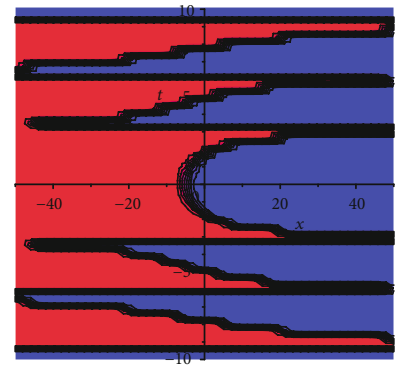

(f3)

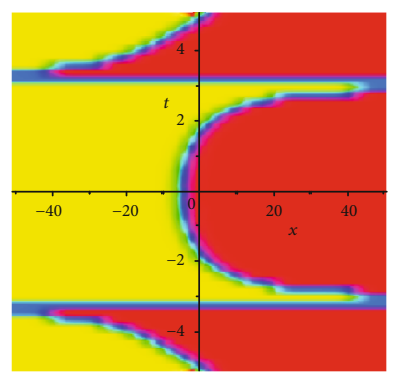

(f4)

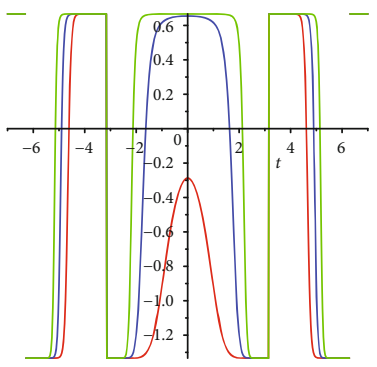

Figure 13: The one-wave solution (62) at $\alpha=0.5, \beta=0.2, \alpha_{1}=1, \beta_{1}=1.5, \sigma_{2}=2, \operatorname{rho}_{1}=2.2, \rho_{2}=3, g(t)=\sin (t), h(t)=\cos (t)$, and $y=1$.

in which $\rho_{1}, \rho_{2}, \sigma_{1}$ and $\sigma_{2}$ are unfound constants. Inserting (60) into equation (6), we gain the following case:

$$
\begin{aligned}
& \rho_{1}=\rho_{1}, \\
& \rho_{2}=\rho_{2}, \\
& \sigma_{1}=-\frac{\rho_{1}\left(2 \alpha_{1} \rho_{2}-\sigma_{2}\right)}{\rho_{2}},
\end{aligned}
$$

$$
\sigma_{2}=\sigma_{2}
$$$$
\lambda_{1}=\frac{\beta_{1}\left(-\alpha_{1}^{4}+\alpha \beta_{1}^{2}-h(t) \alpha_{1}^{2}\right)}{2 \beta g(t) \alpha_{1}^{2}} .
$$

Therefore, the resulting one-wave solution reads as

$$
\Psi(x, y, t)=\frac{-\left(\rho_{1}\left(2 \alpha_{1} \rho_{2}-\sigma_{2}\right) / \rho_{2}\right)+\sigma_{2} e^{\alpha_{1} x+\beta_{1} y-\left(\beta_{1}\left(-\alpha_{1}^{4}+\alpha \beta_{1}^{2}-h(t) \alpha_{1}^{2}\right) / 2 \beta g(t) \alpha_{1}^{2}\right) t}}{\rho_{1}+\rho_{2} e^{\alpha_{1} x+\beta_{1} y-\left(\beta_{1}\left(-\alpha_{1}^{4}+\alpha \beta_{1}^{2}-h(t) \alpha_{1}^{2}\right) / 2 \beta g(t) \alpha_{1}^{2}\right) t}} .
$$

By choosing the suitable values of parameters, the graphic representation of the periodic wave solution is presented in Figure 13 containing the 3D plot, the contour plot, the density plot, and the $2 \mathrm{D}$ plot when three spaces arise at spaces $x=-5, x=0$, and $x=5$.

7.2. Set II: Two-Wave Solutions. We begin with two-wave functions based on the statement in Step 2 in the previous section; we suppose that equation (6) has the rational function of two-wave solutions as shown in the following form:

$\Psi(x, y, z, t)=\frac{\Delta_{2}}{\Omega_{2}}$,

$$
\begin{aligned}
\Omega_{2}=1 & +\sigma_{1} e^{\alpha_{1} x+\beta_{1} y-\lambda_{1} t}+\sigma_{2} e^{\alpha_{2} x+\beta_{2} y-\lambda_{2} t} \\
& +\sigma_{1} \sigma_{2} \sigma_{12} e^{\left(\alpha_{1}+\alpha_{2}\right) x+\left(\beta_{1}+\beta_{2}\right) y+\left(\gamma_{1}-\left(\lambda_{1}+\lambda_{2}\right) t\right.}, \\
\Delta_{2}= & \rho_{1} e^{\alpha_{1} x+\beta_{1} y-\lambda_{1} t}+\rho_{2} e^{\alpha_{2} x+\beta_{2} y-\lambda_{2} t} \\
& +\rho_{1} \rho_{2} \rho_{12} e^{\left(\alpha_{1}+\alpha_{2}\right) x+\left(\beta_{1}+\beta_{2}\right) y-\left(\lambda_{1}+\lambda_{2}\right) t} .
\end{aligned}
$$

Inserting (63) along with (64) into equation (6), we obtain the following case:

$$
\begin{aligned}
\sigma_{i} & =\sigma_{i}, \\
\lambda_{i} & =\frac{\beta_{i}\left(-\alpha_{i}^{4}+\alpha \beta_{i}^{2}-h(t) \alpha_{i}^{2}\right)}{2 \beta g(t) \alpha_{i}^{2}}, \\
\rho_{i} & =2 \alpha_{i} \sigma_{i}, \\
i & =1,2 .
\end{aligned}
$$

$$
\begin{aligned}
\rho_{12} & =\frac{1}{2} \frac{\alpha_{1}^{2} \alpha_{2}^{2}\left(\alpha_{1}+\alpha_{2}\right)\left(-\alpha_{2}+\alpha_{1}\right)^{2}+\alpha\left(\alpha_{1}+\alpha_{2}\right)\left(\alpha_{1} \beta_{2}-\alpha_{2} \beta_{1}\right)^{2}}{\alpha_{2} \alpha_{1}\left(\alpha_{1}^{2} \alpha_{2}^{2}\left(\alpha_{1}+\alpha_{2}\right)^{2}+\alpha\left(\alpha_{1} \beta_{2}-\alpha_{2} \beta_{1}\right)^{2}\right)}, \\
\sigma_{12} & =\frac{\alpha_{1}^{2} \alpha_{2}^{2}\left(-\alpha_{2}+\alpha_{1}\right)^{2}+\alpha\left(\alpha_{1} \beta_{2}-\alpha_{2} \beta_{1}\right)^{2}}{\alpha_{1}^{2} \alpha_{2}^{2}\left(\alpha_{1}+\alpha_{2}\right)^{2}+\alpha\left(\alpha_{1} \beta_{2}-\alpha_{2} \beta_{1}\right)^{2}} .
\end{aligned}
$$

Therefore, the resulting two-wave solution reads as

$$
\Psi_{2}(x, y, t)=\frac{\sigma_{1} e^{\Lambda_{1}}+\sigma_{2} e^{\Lambda_{2}}+\sigma_{1} \sigma_{2} \sigma_{12} e^{\Lambda_{1}+\Lambda_{2}}}{\rho_{1} e^{\Lambda_{1}}+\rho_{2} e^{\Lambda_{2}}+4 \alpha_{1} \alpha_{2} \sigma_{1} \sigma_{2} \rho_{12} e^{\Lambda_{1}+\Lambda_{2}}},
$$

in which $\Lambda_{i}=\alpha_{i} x+\beta_{i} y-\left(\beta_{i}\left(-\alpha_{i}^{4}+\alpha \beta_{i}^{2}-h(t) \alpha_{i}^{2}\right) / 2 \beta g(t) \alpha_{i}^{2}\right)$ $t, i=1,2$. By choosing the suitable values of parameters, the graphic representation of the periodic wave solution is presented in Figure 14 containing the 3D plot, the contour plot, 
(f1)

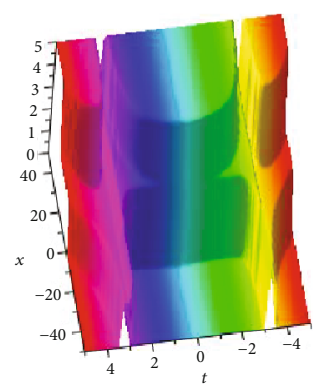

(f2)

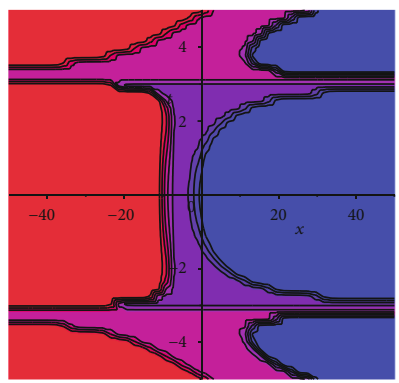

(f3)

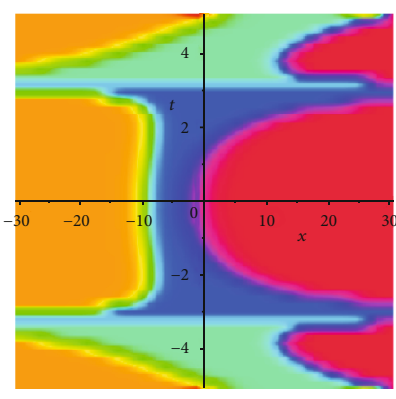

(f4)

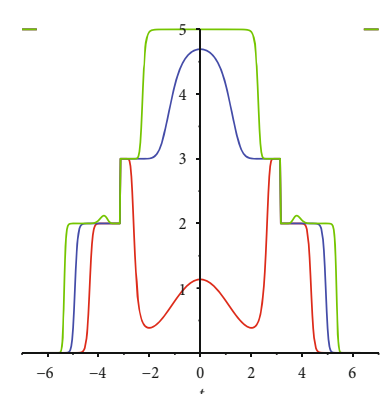

Figure 14: The two-wave solution (67) at $\alpha=0.5, \beta=0.2, \alpha_{1}=1, \alpha_{2}=1.5, \beta_{1}=1.5, \beta_{2}=2, \sigma_{1}=1.1, \sigma_{2}=2, \rho_{1}=2.2, \rho_{2}=3, g(t)=\sin (t)$, $h(t)=\cos (t)$, and $y=1$.

(f1)

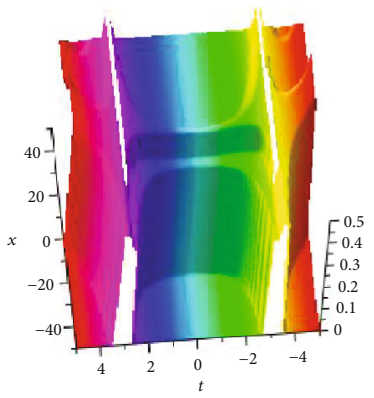

(f2)

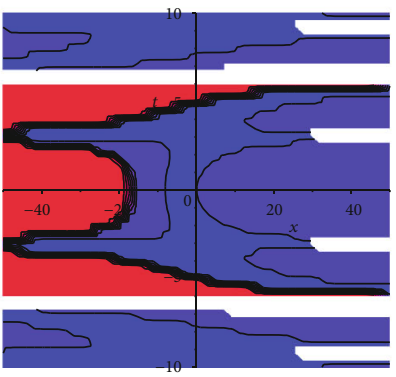

(f3)

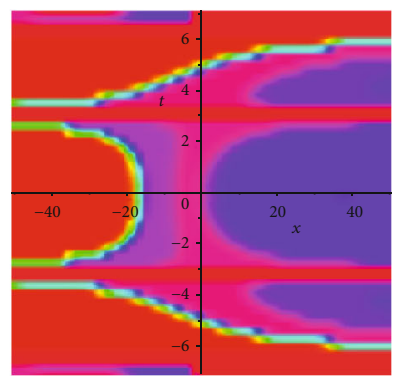

(f4)

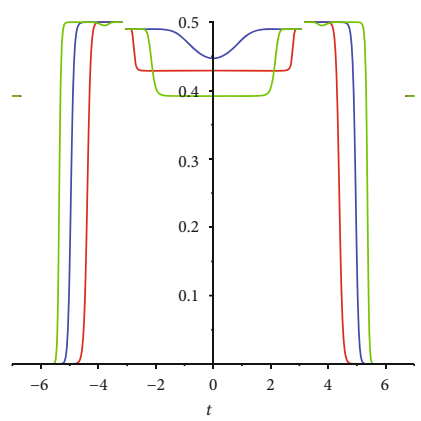

Figure 15: The three-wave solution (71) at $\alpha=0.5, \beta=0.2, \alpha_{1}=1, \alpha_{2}=1.5, \alpha_{3}=2, \beta_{1}=1.5, \beta_{2}=2, \beta_{3}=3.2, \sigma_{1}=1.1, \sigma_{2}=2, \sigma_{3}=1.5, \rho_{1}=$ 2.2, $\rho_{2}=3, g(t)=\sin (t), h(t)=\cos (t)$, and $y=1$.

the density plot, and the $2 \mathrm{D}$ plot when three spaces arise at spaces $x=-10, x=0$, and $x=10$.

7.3. Set III: Triple-Wave Solutions. We commence with threewave functions based on the statement in Step 2 in the previous section; we suppose that equation (6) has the rational function of triple-wave solutions as shown in the following form:

$$
\Psi(x, y, z, t)=\frac{\Delta_{3}}{\Omega_{3}},
$$

$$
\begin{aligned}
\Omega_{3}=1 & +\rho_{1} e^{\Lambda_{1}}+\rho_{2} e^{\Lambda_{2}}+\rho_{3} e^{\Lambda_{3}}+\rho_{1} \rho_{2} \rho_{12} e^{\Lambda_{1}+\Lambda_{2}} \\
& +\rho_{1} \rho_{3} \rho_{13} e^{\Lambda_{1}+\Lambda_{3}}+\rho_{2} \rho_{3} \rho_{23} e^{\Lambda_{2}+\Lambda_{3}} \\
& +\rho_{1} \rho_{2} \rho_{3} \rho_{12} \rho_{13} \rho_{23} e^{\Lambda_{1}+\Lambda_{2}+\Lambda_{3}}, \\
\Delta_{3}= & \sigma_{1} e^{\Lambda_{1}}+\sigma_{2} e^{\Lambda_{2}}+\sigma_{3} e^{\Lambda_{3}}+\sigma_{1} \sigma_{2} \sigma_{12} e^{\Lambda_{1}+\Lambda_{2}} \\
& +\sigma_{1} \sigma_{3} \sigma_{13} e^{\Lambda_{1}+\Lambda_{3}}+\sigma_{2} \sigma_{3} \sigma_{23} e^{\Lambda_{2}+\Lambda_{3}} \\
& +\sigma_{1} \sigma_{2} \sigma_{3} \sigma_{12} \sigma_{13} \sigma_{23} e^{\Lambda_{1}+\Lambda_{2}+\Lambda_{3}},
\end{aligned}
$$

in which $\Lambda_{i}=\alpha_{i} x+\beta_{i} y-\lambda_{i} t, i=1,2,3$. Plugging (68) along with (69) into equation (6), we obtain the following case:

$$
\begin{gathered}
\sigma_{i}=\sigma_{i}, \\
\lambda_{i}=\frac{\beta_{i}\left(-\alpha_{i}^{4}+\alpha \beta_{i}^{2}-h(t) \alpha_{i}^{2}\right)}{2 \beta g(t) \alpha_{i}^{2}}, \\
\rho_{i}=2 \alpha_{i} \sigma_{i}, \\
\quad i=1,2,3, \\
\rho_{12}=\frac{1}{2} \frac{\alpha_{1}^{2} \alpha_{2}^{2}\left(\alpha_{1}+\alpha_{2}\right)\left(-\alpha_{2}+\alpha_{1}\right)^{2}+\alpha\left(\alpha_{1}+\alpha_{2}\right)\left(\alpha_{1} \beta_{2}-\alpha_{2} \beta_{1}\right)^{2}}{\alpha_{2} \alpha_{1}\left(\alpha_{1}^{2} \alpha_{2}^{2}\left(\alpha_{1}+\alpha_{2}\right)^{2}+\alpha\left(\alpha_{1} \beta_{2}-\alpha_{2} \beta_{1}\right)^{2}\right)}, \\
\sigma_{12}=\frac{\alpha_{1}^{2} \alpha_{2}^{2}\left(-\alpha_{2}+\alpha_{1}\right)^{2}+\alpha\left(\alpha_{1} \beta_{2}-\alpha_{2} \beta_{1}\right)^{2}}{\alpha_{1}^{2} \alpha_{2}^{2}\left(\alpha_{1}+\alpha_{2}\right)^{2}+\alpha\left(\alpha_{1} \beta_{2}-\alpha_{2} \beta_{1}\right)^{2}}, \\
\rho_{13}=\text { substitute }\left\{\alpha_{2}=\alpha_{3}, \beta_{2}=\beta_{3}\right\}, \text { at } \rho_{12}, \\
\sigma_{13}=\text { substitute }\left\{\alpha_{2}=\alpha_{3}, \beta_{2}=\beta_{3}\right\}, \text { at } \sigma_{12}, \\
\rho_{23}=\text { substitute }\left\{\alpha_{1}=\alpha_{2}, \beta_{1}=\beta_{2}\right\}, \text { at } \rho_{13}, \\
\sigma_{23}=\text { substitute }\left\{\alpha_{1}=\alpha_{2}, \beta_{1}=\beta_{2}\right\}, \text { at } \sigma_{13} .
\end{gathered}
$$

Therefore, the resulting three-wave solution reads as

$$
\Psi_{3}(x, y, z, t)=\frac{\Delta_{3}}{\Omega_{3}} .
$$

In (71), $\Delta_{3}$ and $\Omega_{3}$ with their relations are given in (69) and (70). By selecting the suitable values of parameters, the 
graphic representation of the periodic wave solution is presented in Figure 15 containing the 3D plot, the contour plot, the density plot, and the $2 \mathrm{D}$ plot when three spaces arise at spaces $x=-10, x=0$, and $x=10$.

This paper finds many novel one-soliton-, two-soliton-, and triple-soliton-type solutions to governing models. With the help of some computations, surfaces of results reported have been observed in Figures 1-15. These figures are dependent on the family conditions which are of importance physically. It has been investigated that all figures plotted have been symbolized for the four types of the nonlinear PDEs containing the extended $(3+1)$-dimensional Jimbo-Miwalike equation, the extended $(2+1)$-dimensional CalogeroBogoyavlenskii-Schiff equation, the generalization of the $(2+1)$-dimensional Bogoyavlensky-Konopelchenko equation, and a variable-coefficient extension of the DJKM equation. These mathematical properties come from exponential function properties. In this sense, from the mathematical and physical points of view, these results play an important role in explaining wave propagation of nonlinear phenomena. Hence, we consider that surfaces plotted in this paper have proven such physical meaning of the obtained solutions.

\section{Conclusion}

In this article, we obtained the multiple soliton solutions of the novel extended $(3+1)$-dimensional Jimbo-Miwalike equation, the extended $(2+1)$-dimensional CalogeroBogoyavlenskii-Schiff equation, the generalization of the $(2+1)$-dimensional Bogoyavlensky-Konopelchenko equation, and a variable-coefficient extension of the DJKM equation via operating the multiple Exp-function method, including one-soliton-, two-soliton-, and triple-soliton-type solutions. It is quite visible that this novel scheme has plenty of family of solutions containing rational exponential functions by selecting particular parameters. Thus, this paper provides a lot of encouragement for future research in soliton topics. The behaviours of the solutions for the known nonlinear equations obtained by the multiple Exp-function method by choosing the suitable values are cited in Figures 1-15. Moreover, the numerical simulations include gaining the coefficients $\rho_{i}, \sigma_{i}$, and $\lambda_{i}$ and have been carried out to show that the projected algorithm is applicable and efficient. The analytical study has been conducted for the solutions $\Psi$ $(x, y, t)$ obtained by employing the aforementioned method, in which obtained solutions were as one-soliton, two-soliton, and triple-soliton. The results are beneficial to the study of wave propagation. All computations in this paper have been made quickly with the aid of Maple.

\section{Data Availability}

The datasets supporting the conclusions of this article are included within the article and its additional file.

\section{Conflicts of Interest}

The authors declare that they have no conflict of interest.

\section{References}

[1] S. H. Seyedi, B. N. Saray, and A. J. Chamkha, "Heat and mass transfer investigation of MHD Eyring-Powell flow in a stretching channel with chemical reactions," Physica A: Statistical Mechanics and its Applications, vol. 544, p. 124109, 2020.

[2] M. Dehghan, J. Manafian, and A. Saadatmandi, "Analytical treatment of some partial differential equations arising in mathematical physics by using the Exp-function method," International Journal of Modern Physics B, vol. 25, pp. 29652981, 2012.

[3] M. Dehghan, J. Manafian, and A. Saadatmandi, "Solving nonlinear fractional partial differential equations using the homotopy analysis method," Numerical Methods for Partial Differential Equations Journal, vol. 26, pp. 448-479, 2010.

[4] M. Dehghan and J. Manafian, "The solution of the variable coefficients fourth-order parabolic partial differential equations by the homotopy perturbation method," Zeitschrift für Naturforschung A, vol. 64, no. 7-8, pp. 420-430, 2009.

[5] J. Manafian and M. Lakestani, "Solitary wave and periodic wave solutions for Burgers, Fisher, Huxley and combined forms of these equations by the $\left(G^{\prime} / G\right)$-expansion method," Pramana, vol. 130, pp. 31-52, 2015.

[6] J. Manafian and M. Lakestani, "Application of $\tan (\phi / 2)$ expansion method for solving the Biswas-Milovic equation for Kerr law nonlinearity," Optik, vol. 127, no. 4, pp. 2040 2054, 2016.

[7] J. Manafian and M. Lakestani, "Dispersive dark optical soliton with Tzitzéica type nonlinear evolution equations arising in nonlinear optics," Optical and Quantum Electronics, vol. 48, no. 2, p. 116, 2016.

[8] A. R. Seadawy and J. Manafian, "New soliton solution to the longitudinal wave equation in a magneto-electro-elastic circular rod," Results in Physics, vol. 8, pp. 1158-1167, 2018.

[9] J. Manafian, "Novel solitary wave solutions for the $(3+1)$ dimensional extended Jimbo-Miwa equations," Computers \& Mathematcs with Applications, vol. 76, no. 5, pp. 1246-1260, 2018.

[10] J. Manafian, B. Mohammadi-Ivatlo, and M. Abapour, "Lumptype solutions and interaction phenomenon to the $(2+1)$ dimensional breaking soliton equation," Applied Mathematics and Computation, vol. 13, pp. 13-41, 2019.

[11] W. X. Ma, Y. Zhou, and R. Dougherty, "Lump-type solutions to nonlinear differential equations derived from generalized bilinear equations," International Journal of Modern Physics $B$, vol. 30, no. 28n29, article 1640018, 2016.

[12] J. Lü, S. Bilige, X. Gao, Y. Bai, and R. Zhang, "Abundant lump solutions and interaction phenomena to the Kadomtsev-Petviashvili-Benjamin-Bona-Mahony equation," Journal of Applied Mathematics and Physics, vol. 6, no. 8, pp. 1733$1747,2018$.

[13] C. Wang, "Spatiotemporal deformation of lump solution to $(2+1)$-dimensional KdV equation," Nonlinear Dynamics, vol. 84, no. 2, pp. 697-702, 2016.

[14] J. H. He, "A modified Li-He's variational principle for plasma," International Journal of Numerical Methods for Heat and Fluid Flow, 2019.

[15] J. H. He, "Lagrange crisis and generalized variational principle for 3D unsteady flow," International Journal of Numerical Methods for Heat and Fluid Flow, vol. 30, no. 3, pp. 11891196, 2019. 
[16] S. S. Chen, B. Tian, L. Liu, Y. Q. Yuan, and C. R. Zhang, "Conservation laws, binary Darboux transformations and solitons for a higher-order nonlinear Schrödinger system," Chaos, Solitons and Fractals, vol. 118, pp. 337-346, 2019.

[17] X. X. Du, B. Tian, X. Y. Wu, H. M. Yin, and C. R. Zhang, "Lie group analysis, analytic solutions and conservation laws of the $(3+1)$-dimensional Zakharov-Kuznetsov-Burgers equation in a collisionless magnetized electron-positron-ion plasma," The European Physical Journal Plus, vol. 133, no. 9, p. 378, 2018.

[18] S. Saha Ray, "On conservation laws by Lie symmetry analysis for $(2+1)$-dimensional BogoyavlenskyKonopelchenko equation in wave propagation," Computers \& Mathematics with Applications, vol. 74, pp. 1158-1165, 2017.

[19] X. H. Zhao, B. Tian, X. Y. Xie, X. Y. Wu, Y. Sun, and Y. J. Guo, "Solitons, Bäcklund transformation and Lax pair for a $(2+1)$ dimensional Davey-Stewartson system on surface waves of finite depth," Wave Random Complex, vol. 28, pp. 356-366, 2017.

[20] H. M. Baskonus and H. Bulut, "Exponential prototype structures for $(2+1)$-dimensional Boiti-Leon-Pempinelli systems in mathematical physics," Waves in Random and Complex Media, vol. 26, pp. 201-208, 2016.

[21] H. M. Baskonus, D. A. Koç, and H. Bulut, "New travelling wave prototypes to the nonlinear Zakharov-Kuznetsov equation with power law nonlinearity," Nonlinear Analysis: Real World Applications, vol. 7, pp. 67-76, 2010.

[22] Q. Zhou, "Optical solitons in medium with parabolic law nonlinearity and higher order dispersion," Waves in Random and Complex Media, vol. 25, pp. 52-59, 2016.

[23] T. A. Sulaiman, R. I. Nuruddeen, E. Zerrad, and B. B. Mikail, "Dark and singular solitons to the two nonlinear Schrödinger equations," Optik, vol. 186, pp. 423-430, 2019.

[24] M. Inc, A. I. Aliyu, A. Yusuf, and D. Baleanu, "Optical solitary waves, conservation laws and modulation instability analysis to the nonlinear Schrödinger's equation in compressional dispersive Alvèn waves," Optik, vol. 155, pp. 257-266, 2018.

[25] M. Jimbo, "Solitons and infinite-dimensional lie algebras," Kyoto University, vol. 19, no. 3, pp. 943-1001, 1983.

[26] W. Tan, Z. D. Dai, J. L. Xie, and L. L. Hu, "Emergence and interaction of the lump-type solution with the $(3+1)$-D JimboMiwa equation," Zeitschrift für Naturforschung, vol. 73, no. 1, pp. 43-49, 2017.

[27] J. Y. Yang and W. X. Ma, "Abundant lump-type solutions of the Jimbo-Miwa equation in $(3+1)$-dimensions," Computers \& Mathematcs with Applications, vol. 73, no. 2, pp. 220-225, 2017.

[28] W. X. Ma, "Lump-type solutions to the $(3+1)$-dimensional Jimbo-Miwa equation," International Journal of Nonlinear Sciences and Numerical Simulation, vol. 17, no. 7-8, p. 355, 2016.

[29] W. X. Ma and J. H. Lee, "A transformed rational function method and exact solutions to the $3+1$ dimensional JimboMiwa equation," Chaos, Solitons \& Fractals, vol. 42, no. 3, pp. 1356-1363, 2009.

[30] Y. N. Tang, W. X. Ma, W. Xu, and L. Gao, "Wronskian determinant solutions of the $(3+1)$-dimensional Jimbo-Miwa equation," Applied Mathematics and Computation, vol. 217, no. 21, pp. 8722-8730, 2011.

[31] S. Batwa and W. X. Ma, "A study of lump-type and interaction solutions to a (3+1)-dimensional Jimbo-Miwa-like equation," Computers \& Mathematcs with Applications, vol. 76, no. 7, pp. 1576-1582, 2018.
[32] F. H. Qi, Y. H. Huang, and P. Wang, "Solitary-wave and new exact solutions for an extended $(3+1)$-dimensional JimboMiwa-like equation," Applied Mathematics Letters, vol. 100, p. 106004, 2020.

[33] H. Q. Sun and A. H. Chen, "Lump and lump-kink solutions of the $(3+1)$-dimensional Jimbo-Miwa and two extended JimboMiwa equations," Applied Mathematics Letters, vol. 68, pp. 5561, 2017.

[34] Y. F. Yue, L. L. Huang, and Y. Chen, "Localized waves and interaction solutions to an extended $(3+1)$-dimensional Jimbo-Miwa equation," Applied Mathematics Letters, vol. 89, pp. 70-77, 2019.

[35] B. Ren, J. Lin, and Z. M. Lou, “A new nonlinear equation with lump-soliton, lump-periodic, and lump-periodic-soliton solutions," Complexity, vol. 2019, Article ID 4072754, 10 pages, 2019.

[36] A. M. Wazwaz, "The $(2+1)$ and $(3+1)$-dimensional CBS equations: multiple soliton solutions and multiple singular soliton solutions," Zeitschrift für Naturforschung A, vol. 65, no. 3, pp. 173-181, 2010.

[37] W. R. Sun, B. Tian, H. L. Zhen, and Y. Sun, "Breathers and rogue waves of the fifth-order nonlinear Schrödinger equation in the Heisenberg ferromagnetic spin chain," Nonlinear Dynamics, vol. 81, no. 1-2, pp. 725-732, 2015.

[38] Y. Zhang and J. Pang, "Lump and lump-type solutions of the generalized $(3+1)$-dimensional variable-coefficient B-type Kadomtsev-Petviashvili equation," Journal of Applied Mathematics, vol. 2019, Article ID 7172860, 5 pages, 2019.

[39] H. Leblond and D. Mihalache, "Models of few optical cycle solitons beyond the slowly varying envelope approximation," Physics Reports, vol. 523, no. 2, pp. 61-126, 2013.

[40] D. Mihalache, "Multidimensional localized structures in optical and matter-wave media: a topical survey of recent literature," Romanian Reports in Physics, vol. 69, p. 403, 2017.

[41] P. A. Clarkson and M. D. Kruskal, "New similarity reductions of the Boussinesq equation," Journal of Mathematical Physics, vol. 30, no. 10, pp. 2201-2213, 1989.

[42] J. Weiss, M. Tabor, and G. Carnevale, "The Painlevé property for partial differential equations," Journal of Mathematical Physics, vol. 24, no. 3, pp. 522-526, 1983.

[43] K. R. Adem and C. M. Khalique, "Exact solutions and conservation laws of Zakharov-Kuznetsov modified equal width equation with power law nonlinearity," Nonlinear Analysis: Real World Applications, vol. 13, no. 4, pp. 1692-1702, 2012.

[44] A. M. Wazwaz, Partial Differential Equations and Solitary Waves Theorem, Springer and HEP, Berlin, 2009.

[45] A. M. Wazwaz, "Negative-order forms for the CalogeroBogoyavlenskii-Schiff equation and the modified CalogeroBogoyavlenskii-Schiff equation," Proceedings of the Romanian Academy. Series A, vol. 18, no. 4, pp. 337-344, 2017.

[46] S. Chen, F. Baronio, J. M. Soto-Crespo, P. Grelu, and D. Mihalache, "Versatile rogue waves in scalar, vector, and multidimensional nonlinear systems," Journal of Physics A, vol. 50, no. 46, p. 463001, 2017.

[47] Y. V. Kartashov, G. E. Astrakharchik, B. A. Malomed, and L. Torner, "Frontiers in multidimensional self-trapping of nonlinear fields and matter," Nature Reviews Physics, vol. 1, no. 3, pp. 185-197, 2019.

[48] A. M. Wazwaz, "A $(2+1)$-dimensional time-dependent DateJimbo-Kashiwara-Miwa equation: Painlevé integrability and 
multiple soliton solutions," Computers \& Mathematcs with Applications, vol. 79, no. 4, pp. 1145-1149, 2020.

[49] W. X. Ma, T. Huang, and Y. Zhang, "A multiple expfunction method for nonlinear differential equations and its application," Physica Scripta, vol. 82, no. 6, article 065003, 2010.

[50] W. X. Ma and Y. Zhou, "Lump solutions to nonlinear partial differential equations via Hirota bilinear forms," Journal of Differential Equations, vol. 264, no. 4, pp. 2633-2659, 2018.

[51] W. X. Ma, "Interaction solutions to Hirota-Satsuma-Ito equation in $(2+1)$-dimensions," Frontiers of Mathematics in China, vol. 14, no. 3, pp. 619-629, 2019.

[52] W. X. Ma, "Lump and interaction solutions to linear PDEs in $2+1$ dimensions via symbolic computation," Modern Physics Letters B, vol. 33, no. 36, p. 1950457, 2019.

[53] A. Cordero, J. P. Jaiswal, and J. R. Torregrosa, "Stability analysis of fourth-order iterative method for finding multiple roots of non-linear equations," Applied Mathematics and Nonlinear Sciences, vol. 4, no. 1, pp. 43-56, 2019.

[54] W. Gao, H. F. Ismael, A. M. Husien, H. Bulut, and H. M. Baskonus, "Optical soliton solutions of the cubic-quartic nonlinear Schrödinger and resonant nonlinear Schrödinger equation with the parabolic law," Applied Sciences, vol. 10, no. 1, p. 219, 2020.

[55] Z. Zhao and B. Han, "Residual symmetry, Bäcklund transformation and CRE solvability of a $(\$ \$ \backslash$ mathbf $\{2\}\{\backslash$ varvec $\{+\}\}\{\}$ Imathbf\{1\}\$\$ 2 + 1)-dimensional nonlinear system," Nonlinear Dynamics, vol. 94, no. 1, pp. 461-474, 2018.

[56] W. Gao, G. Yel, H. M. Baskonus, and C. Cattani, "Complex solitons in the conformable $(2+1)$-dimensional AblowitzKaup-Newell-Segur equation," Aims Mathematics, vol. 5, no. 1, pp. 507-521, 2020.

[57] W. Gao, H. Rezazadeh, Z. Pinar, H. M. Baskonus, S. Sarwar, and G. Yel, "Novel explicit solutions for the nonlinear Zoomeron equation by using newly extended direct algebraic technique," Optical and Quantum Electronics, vol. 52, no. 52, pp. 1-13, 2020.

[58] W. Gao, H. F. Ismael, H. Bulut, and H. M. Baskonus, "Instability modulation for the $(2+1)$-dimension paraxial wave equation and its new optical soliton solutions in Kerr media," Physica Scripta, vol. 95, no. 35207, pp. 1-12, 2020.

[59] W. Gao, B. Ghanbari, H. Günerhan, and H. M. Baskonus, "Some mixed trigonometric complex soliton solutions to the perturbed nonlinear Schrödinger equation," Modern Physics Letters B, vol. 34, no. 3, article 2050034, 2020.

[60] Y. Yakup and Y. Emrullah, "Multiple exp-function method for soliton solutions of nonlinear evolution equations," Chinese Physics B, vol. 26, no. 7, pp. 20-26, 2017.

[61] A. G. Alnowehy, "The multiple exp-function method and the linear superposition principle for solving the $(2+1)$-dimensional Calogero-Bogoyavlenskii-Schiff equation," Zeitschrift für Naturforschung A, vol. 70, no. 9, pp. 775-779, 2015.

[62] R. A. Abdullahi, "The generalized $(1+1)$-dimensional and $(2+1)$-dimensional Ito equations: multiple exp-function algorithm and multiple wave solutions," Computers \& Mathematcs with Applications, vol. 71, pp. 1248-1258, 2016.

[63] Y. Long, Y. He, and S. Li, "Multiple soliton solutions for a new generalization of the associated Camassa-Holm equation by exp-function method," Mathematical Problems in Engineering, vol. 2014, 7 pages, 2014.
[64] W. X. Ma and Z. Zhu, "Solving the $(3+1)$-dimensional generalized KP and BKP equations by the multiple exp-function algorithm," Applied Mathematics and Computation, vol. 218, no. 24, pp. 11871-11879, 2012.

[65] J. G. Liu, L. Zhou, and Y. He, "Multiple soliton solutions for the new $(2+1)$-dimensional Korteweg-de Vries equation by multiple exp-function method," Applied Mathematics Letters, vol. 80, pp. 71-78, 2018. 